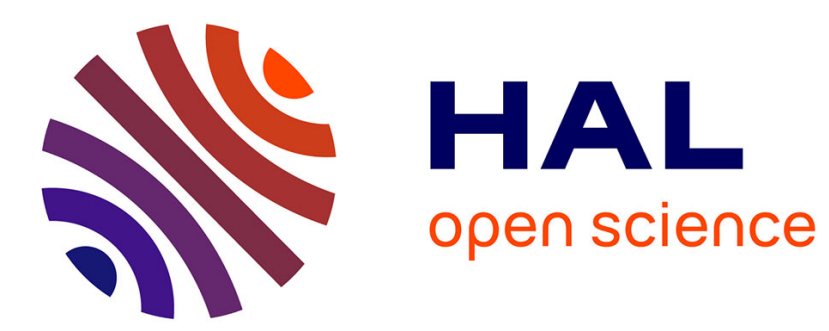

\title{
Maintenance and investment: Complements or substitutes? A reappraisal
}

\author{
R. Boucekkine, G. Fabbri, F. Gozzi
}

\section{To cite this version:}

R. Boucekkine, G. Fabbri, F. Gozzi. Maintenance and investment: Complements or substitutes? A reappraisal. Journal of Economic Dynamics and Control, 2010, 34 (12), pp.2420. 10.1016/j.jedc.2010.06.007 . hal-00743831

\section{HAL Id: hal-00743831 \\ https://hal.science/hal-00743831}

Submitted on 21 Oct 2012

HAL is a multi-disciplinary open access archive for the deposit and dissemination of scientific research documents, whether they are published or not. The documents may come from teaching and research institutions in France or abroad, or from public or private research centers.
L'archive ouverte pluridisciplinaire HAL, est destinée au dépôt et à la diffusion de documents scientifiques de niveau recherche, publiés ou non, émanant des établissements d'enseignement et de recherche français ou étrangers, des laboratoires publics ou privés. 


\section{Author's Accepted Manuscript}

Maintenance and investment: Complements or substitutes? A reappraisal

R. Boucekkine, G. Fabbri, F. Gozzi

PII:

S0165-1889(10)00137-5

DOI: doi:10.1016/j.jedc.2010.06.007

Reference: DYNCON 2437

To appear in: Journal of Economic Dynamics

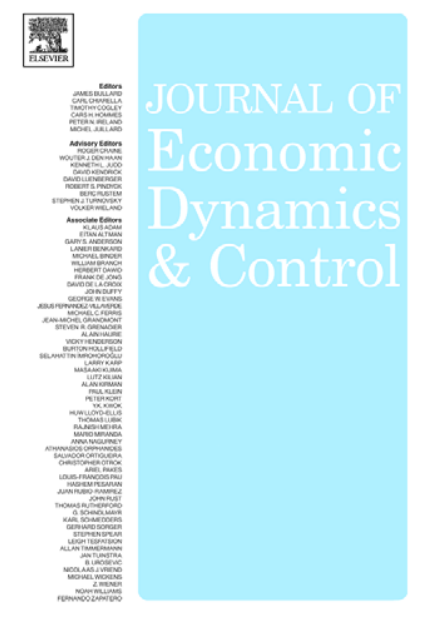

www.elsevier.com/locate/jedc \& Control

Received date: 17 September 2009

Accepted date: 1 June 2010

Cite this article as: R. Boucekkine, G. Fabbri and F. Gozzi, Maintenance and investment: Complements or substitutes? A reappraisal, Journal of Economic Dynamics \& Control, doi:10.1016/j.jedc.2010.06.007

This is a PDF file of an unedited manuscript that has been accepted for publication. As a service to our customers we are providing this early version of the manuscript. The manuscript will undergo copyediting, typesetting, and review of the resulting galley proof before it is published in its final citable form. Please note that during the production process errors may be discovered which could affect the content, and all legal disclaimers that apply to the journal pertain. 


\title{
Maintenance and Investment: Complements or Substitutes? A Reappraisal ${ }^{\text {tर }}$
}

\author{
R. Boucekkine ${ }^{\mathrm{a}, 1, *}$, G. Fabbri ${ }^{\mathrm{b}}$, F.Gozzi $^{\mathrm{c}}$ \\ ${ }^{a}$ Department of Economics and CORE, Place Montesquieu 3, Université catholique de Louvain, \\ Louvain-La-Neuve, Belgium; Department of Economics, University of Glasgow. \\ ${ }^{b}$ Dipartimento di Studi Economici S. Vinci, Università di Napoli "Parthenope", Naples, Italy. \\ ${ }^{c}$ Dipartimento di Scienze Economiche ed Aziendali, Università LUISS - Guido Carli Rome, Italy.
}

\begin{abstract}
A benchmark AK optimal growth model with maintenance expenditures and endogenous utilization of capital is considered within an explicit vintage capital framework. Scrapping is endogenous, and the model allows for a clean distinction between age and usage dependent capital depreciation and obsolescence. It is also shown that in this set-up past investment profile completely determines the size of current maintenance expenditures. Among other findings, a closed-form solution to optimal dynamics is provided taking advantage of very recent development in optimal control of infinite dimensional systems. More importantly, and in contrast to the pre-existing literature, we study investment and maintenance co-movements without any postulated ad-hoc depreciation function. In particular using impulse response experiments, we find that optimal investment and maintenance do move together in the short-run in response to neutral technological shocks, which seems to be more consistent with the data.
\end{abstract}

Keywords: Maintenance, Investment, optimal control, dynamic programming, infinite dimensional problem.

JEL classification: E22, E32, O40

\section{Introduction}

There is an increasing effort to incorporate maintenance and repair activities in the core of investment theory, and therefore in the core of growth theory. While traditional Jorgensonian investment theory relies on the assumption of constant capital depreciation rate, several authors have been pointing at the numerous shortcomings arising from such an assumption, therefore challenging the ability of the standard model to account for the

\footnotetext{
We would like to thank two anonymous referees for constructive feedback. We have also benefited from several useful comments by Fernando del Río, Blanca Martinez, Omar Licandro, Luis Puch and Ramón Ruiz-Tamarit. Of course, any remaining error is ours.

* Corresponding author

Email addresses: raouf.boucekkine@uclouvain.be (R. Boucekkine), giorgio.fabbri@uniparthneope.it (G. Fabbri), fgozzi@luiss.it (F.Gozzi)

${ }^{1}$ Boucekkine acknowledges support from the ARC project 09/14-018 on Sustainability.

Preprint submitted to Journal of Economic Dynamics and Control

June 1, 2010
} 
investment decision either at the firm or the aggregate levels. Feldstein and Rothschild (1974) and Nickell (1975) are two pioneering works in this respect (see also Hartl, 1983, for a more technical early contribution to the literature).

Yet the incorporation of maintenance and repair costs in macroeconomic models of investment and growth has truly started with the illuminating work of McGrattan and Schmitz (1999). Indeed the lack of economy-wide surveys assessing the importance of maintenance costs was usually invoked to disregard them. McGrattan and Schmitz were the first to exploit a Canadian (economy-wide) survey, and to highlight why and how investment theory can account for these costs. Their argument was actually easy to make as maintenance and repair expenditures averaged about $6 \%$ of the Canadian GDP and nearly $50 \%$ of spending on new equipment over the period 1961-1993.

Since then, several research projects have been launched on the topic. Let us mention briefly some of the directions taken. A first bulk of papers studied the implications of accounting for maintenance expenditures within otherwise standard models of investment and/or growth. For example Licandro et al. (2001) introduced maintenance in optimal growth by specifying capital depreciation as a decreasing function of maintenance. They studied accordingly how the latter affects the typical convergence properties inherent to neoclassical theory. Boucekkine and Ruiz-Tamarit (2003) took a tighter avenue, the standard neoclassical investment firm model, ${ }^{2}$ but they allowed for a more flexible relationship between depreciation, maintenance expenditures and the rate of capital utilization. They showed that depending on some deep characteristics of the latter relationship, investment and maintenance decisions can respond (or not) in the same way to various neutral technological shocks. Co-movements of investment and maintenance are the central motivation of our paper, so we will come back later to this point. Note however that the previous papers only provide characterizations around steady state equilibria, while our paper solves out the short-term optimal dynamics. On the quantitative macroeconomics ground, more papers have been written recently. ${ }^{3}$ Important contributions to this line of research are due to Kalaitzidakis and Kalyvitis $(2004,2005)$ who studied how maintenance of public capital affects long-term growth and how to fix optimally maintenance expenditures in this respect.

More recently, Saglam and Veliov (2008), Goetz, Hritonenko and Yatsenko (2008) and Boucekkine et al. (2009) have shown how to incorporate maintenance and support costs in vintage capital models. The idea that maintenance costs do depend on the age of capital goods is natural in many respects, especially if one has to account for technological obsolescence in addition to physical deterioration. While incorporating explicitly maintenance and operation costs, Goetz et al. (2008) have focused more on the derivation of optimal investment and disinvestment rules. Saglam and Veliov (2008) and Boucekkine et al. (2009) have paid much more attention to the co-movements between investment and maintenance, the former in a firm problem and the latter in a general equilibrium set-up. Other differences between the two frameworks lie in the fact that while the former

\footnotetext{
${ }^{2}$ Another paper taking this avenue is Kalyvitis (2006) who empirically found that the incorporation of maintenance and repair expenditures into the q-model of investment improves the relevance of the latter.

${ }^{3}$ An early investigation of the role of maintenance in the business cycle is due to Collard and Kollintzas (2000).
} 
(as almost all the other papers listed so far) builds on a given functional form directly relating maintenance to depreciation, and considers maintenance as a control variable (as initiated by McGrattan and Schmitz (1999)), ${ }^{4}$ the latter derives depreciation partly as a result of the endogenous scrapping decision of obsolete capital items, and specifies maintenance costs as a function of the chosen capacity utilization and the age of capital goods. Therefore the main advantage of the latter approach is to allow for an endogenous identification of depreciation due to obsolescence on one hand, and depreciation due to aging and utilization on the other.

Boucekkine et al. (2009) consider a multi-sector model with both investment-specific and neutral technological progress, and Cobb-Douglas production function in the final goods sector. In this paper, we shall consider a much simpler AK one-sector version of Boucekkine et al. (2009), only allowing for neutral technological progress. Moreover, while these authors focus on the steady state, we are able to:

1. find a closed-form solution to optimal dynamics by applying the dynamic programming strategy developed by Fabbri and Gozzi (2008a);

2. and accordingly to study how optimal investment and maintenance move in response to technological shocks, not only in the long-run, but also in the short-run.

In this sense, our paper has a double technical contribution: on one hand, it derives a closed-form solution to the optimal dynamics in the vintage AK version of Boucekkine et al. (2009), and on the other, it shows how to apply the dynamic programming approach of Fabbri and Gozzi to an AK model with endogenous scrapping. The first application, provided by the authors themselves, was on the AK vintage capital model of Boucekkine et al. (2005) which has exogenous scrapping time. Because the involved optimal control problem has a differential-difference state equation, the problem is infinite dimensional, and the dynamics cannot be studied with the standard techniques. More precisely, applying a Pontryagin-type argument is still possible as demonstrated in Boucekkine et al. (2005) but the stability analysis is much more sophisticated and requires nonstandard tools. The main advantage of the dynamic programming approach applied in this paper is to deliver a simple explicit value function, which allows to visualize the optimal dynamics straightforwardly, which is not possible within the Pontryagin-based approach. ${ }^{5}$

More importantly, our paper brings out an original contribution to an important question in the literature, investment and maintenance co-movements. In the earlier papers devoted to this issue, notably those devoted to the study of business fluctuations (see Collard and Kollintzas (2000) and specially Licandro and Puch (2000)), the specification of the depreciation function was chosen in order to replicate some key stylized facts. As mentioned above, Saglam and Veliov (2008) is one of the very few general inspections into this issue. To make our argument more precise, whence the depreciation function

\footnotetext{
${ }^{4}$ Saglam and Veliov (2008) and, to a certain extent, Boucekkine and Ruiz-Tamarit (2003) are among the very few papers considering general depreciation functions: these functions are usually fully specified in the literature, notably the business cycle fluctuations literature.

${ }^{5}$ Other advantages of the dynamic programming approach are given in Fabbri and Gozzi (2008a) (like the less stringent parametric conditions needed). Of course, it should be clear that these advantages are specific to the vintage AK models considered where an explicit value function can be identified.
} 
$\delta(U, M)$ is postulated, with $U$ the capital utilization rate and $M$ maintenance expenditures, assumptions on second-order derivatives of function $\delta(U, M)$ are typically made to fit the data. In particular, it turned out that the sign of the second-order cross derivative $\delta_{U M}(U, M)$ is crucial for the shape of investment and maintenance co-movements. As mentioned above, Boucekkine and Ruiz-Tamarit (2003) have provided with a systematic analysis of the problem depending on the sign of this cross-derivative. When $\delta_{U M}(U, M) \geq 0$, then investment and maintenance move in the same direction (Proposition 3, Boucekkine and Ruiz-Tamarit, 2003). This case is considered to be compatible with data by Licandro and Puch (2000). However, when $\delta_{U M}(U, M)<0$, things are much trickier, and under certain circumstances, maintenance can act as a substitute to investment, as previously claimed by McGrattan and Schmitz (1999). Our paper offers a sounder framework to study these co-movements. First of all, in contrast to Boucekkine and Ruiz-Tamarit (2003), we leave the steady state and address the problem along optimal transitional dynamics. Second of all, there is no postulated depreciation function, and the depreciation rate is identified in particular through an endogenous scrapping decision. Our main result is that in such a framework, optimal investment and maintenance do move together in the short-run in response to neutral technological shocks. Our results are consistent with those gathered by Saglam and Veliov (2008) in their firm problem who also show analytically that maintenance and investment in new capital goods appear as complements with respect to changes in productivity. ${ }^{6}$

The paper is organized as follows. Section 2 gives a brief description of the AK vintage model and presents the infinite dimensional optimal control problem under consideration. Section 3 recalls the principles of the dynamic programming approach used to solve it, and proceeds further by deriving closed-form solutions to optimal dynamics. Section 4 compiles some numerical exercises conducted to study optimal investment and maintenance co-movements. Section 5 concludes.

\section{The model}

The model is one-sector AK optimal growth model with vintage capital. A benevolent central planner maximizes a discounted intertemporal stream of utilities from consumption under technological and resource constraints. To present things in the simplest way, we first get through the technological specifications. In particular, we will identify the different determinants of capital depreciation in our set-up, and the resulting capital accumulation over time. Then we present the optimal growth model under the extracted capital law of motion.

\subsection{Maintenance, utilization and the sources of capital depreciation}

The aggregate production function is $A K$ :

$$
Y(t)=A k_{t}, A>0
$$

where $k_{t}$ is the stock of capital at time $t$, which is given by

\footnotetext{
${ }^{6}$ Saglam and Veliov (2008) have also derived interesting results on the co-movements between investment in old vintages and maintenance activities. In our model, the economy only invests in new capital goods.
} 


$$
k_{t}=\int_{t-T(t)}^{t} x(z, t) i(z) d z
$$

where $i(z)$ is investment at time $z$, and $T(t)$ is the age of the oldest machine still in use at time $t$ or scrapping time. Both $x(z, t)$ and $T(t)$ are key decision variables in the model. As in Boucekkine et al. (2009), $x(z, t)$ is not an utilization rate, it rather measures the frequency at which the capital good is used (like the number of kilometers run measures the frequency of use of a car). The larger $x(z, t)$, the larger will be the contribution of machines $i(z)$ to output. The scrapping time, $T(t)$, is chosen in such way that non-profitable capital goods at time $t$ are immediately thrown out, as usual in the vintage literature. As we will see in a minute, the determination of optimal $x(z, t)$ and $T(t)$ does not involve an intertemporal trade-off in our model, so we treat them just below independently of the optimal control problem. Before getting to this point, notice that in contrast to Boucekkine et al. (2009), the unique (exogenous) technical progress indicator in the production function is parameter $A$, which may reflect the state of disembodied (neutral) technological change in the sense that all operating machines are affected in the same way by technological accelerations through $A$. By taking this avenue, comparison with more standard RBC approaches like Licandro and Puch (2000) is more appropriate.

Let us come now to the optimal determination of $x(z, t)$ and $T(t)$. It is tightly linked to a crucial ingredient of our model: maintenance and repair expenditures. Regarding this specification aspect, we depart clearly from the literature, including Saglam and Veliov (2008), and we extend a previous specification of Whelan (2002) by assuming a variable maintenance cost. ${ }^{7}$ More specifically, the unit maintenance and repair cost of vintage $z$ at time $t$ is assumed to be an increasing function of its age, $t-z$, and its relative average productivity,

$$
\omega(t-z, x(z, t))=\beta e^{\gamma(t-z)} x(z, t)^{\mu}+\eta, \quad \gamma>0, \beta>0, \eta>0, \mu>1 .
$$

When $\beta=0$, we get Whelan's specification, maintenance costs become equal to a constant support cost. Notice that the specification is completely generic: beside aging, maintenance costs increase with utilization. $\mu>1$ and $\eta>0$ are needed to have a finite optimal choice of utilization and for a finite time scrapping to be possible respectively. Indeed, production net of the maintenance and repair costs is

where

$$
\begin{gathered}
y(t)=\int_{t-T(t)}^{t} B(t-z, x(z, t)) i(z) d z, \\
B(t-z, x(z, t))=A x(z, t)-\omega(t-z, x(z, t))
\end{gathered}
$$

is the average (and marginal) profitability of vintage $z$ at time $t$. The utilization intensities $x(z, t)$ are chosen to maximize the average profitability, $B(t-z, x(z, t))$. The first order condition of this maximization problem implies that $x(z, t)$ depends only on the difference $t-z$ and

$$
x(t-z)=x_{0} e^{-\frac{\gamma}{\mu-1}(t-z)} .
$$

\footnotetext{
${ }^{7}$ Whelan (2002) considered a constant support cost for any vintage at any time.
} 
where $x_{0}=\left(\frac{A}{\beta \mu}\right)^{\frac{1}{\mu-1}}$, inducing a decreasing age profile for utilization. The profile is shifted upward by neutral technological improvements through parameter $A$, and shifted down if the maintenance technology worsens through parameters $\beta$ and $\gamma$.

Finally a machine of vintage $z$ is scrapped whence its profitability drops to zero. Substitution from $x(t-z)$ into (3) yields:

$$
B(t-z) \equiv B(t-z, x(t-z))=\Omega e^{-\frac{\gamma}{\mu-1}(t-z)}-\eta,
$$

where $\Omega=(\mu-1) \beta x_{0}^{\mu}$, which implies that a vintage will be utilized until a finite age $T>0$ such that

$$
B(T)=\Omega e^{-\frac{\gamma}{\mu-1} T}-\eta=0 .
$$

It is easy to prove that whence $\Omega>\eta>0$, a finite (and positive) scrapping time exists and is unique: $T=\frac{\mu-1}{\gamma} \ln \frac{\Omega}{\eta}$. Notice that the model degenerates into a standard AK model if $\eta$ goes to zero. In such a case, $T=\infty$. We can still work out the model and study maintenance and investment co-movements in such a simplistic model but of course we lose an important ingredient: obsolescence. It is also straightforward to see that $T$ is an increasing function of $A$ and a decreasing function of $\beta, \gamma$ and $\eta$. Technological improvement through $A$ increases the profitability of ALL vintages, leading to lengthening their lifetime. Notice that this property arises because technological progress is neutral in the latter case. Investment-specific technological progress would lead to shorter lifetimes, see Boucekkine et al. (2009). Here, since we are interested in investment and maintenance co-movements, and mainly for comparison with the results gathered by Licandro and Puch (2000) or Boucekkine and Ruiz-Tamarit (2003) for example, we consider neutral technological progress accelerations in a one-sector model. ${ }^{8}$

Finally and as announced in the introduction, our model allows to disentangle obsolescence and physical depreciation in a quite easy and natural way. The stock of capital at time $t$ can vary due to (i) gross investment, (ii) the change of the relative average productivity of capital, which is physical depreciation, and (iii) the scrapping of unprofitable vintages, which is called obsolescence. Differentiating equation (1), and using that $x(t-z)$ is given by (4), yields the following evolution law of capital:

$$
\dot{k}(t)=x_{0} i(t)-(\xi(t)+\delta) k(t)
$$

where

$$
\xi(t)=x_{0} e^{-\frac{\gamma}{\mu-1} T} \frac{i(t-T)}{k(t)}
$$

is related to the fraction of scrapped capital at time $t$, and

$$
\delta=\frac{\gamma}{\mu-1}
$$

is the decline rate of the average relative productivity or equivalently utilization intensity of each vintage. For quite intuitive reasons, we shall call $\xi(t)$ the rate of obsolescence, and the constant $\delta$ the rate of physical depreciation since it is related to aging and usage.

\footnotetext{
${ }^{8}$ Needless to say, considering investment-specific technological progress requires at least two sectors, consumption and capital sectors. See Boucekkine et al. (2009).
} 
There is a big difference between the behavior of the obsolescence rate and the physical depreciation rate. While the physical depreciation rate is constant, the obsolescence rate is not so because it depends on the scrapped investment-capital ratio and it could fluctuate due to the existence of echo effects that generally characterize the dynamics of vintage capital models as proved by Boucekkine et al. (2005). On the other hand, and more importantly for our paper, the rate of physical depreciation need not respond in the same way to technological accelerations or other shocks. For example, if $A$ increases, the rate $\delta$ is unaffected while the obsolescence rate $\xi(t)$ is likely to respond given that the impact of $A$ 's increase on lifetime $T$ does affect $\xi(t)$ in several ways. We shall come back to these questions later.

We are ready now to write down the optimal growth problem to be tackled.

\subsection{The optimal growth problem}

The optimal growth problem is standard in that it consists in maximizing intertemporal utility subject to the AK production technology. The per-period utility function is CARA (with $\frac{1}{\sigma}, \sigma>0$, the elasticity of intertemporal substitution in consumption), and an interesting feature of the approach is the possibility to save one control variable, maintenance, by using the net production function (2). Needless to say, capital, maintenance and replacement investment can be reconstructed from the computed controls and states. Indeed, these three variables, $k(\cdot), M(t)$ and $i_{r}(\cdot)$ respectively, are determined by:

and

$$
\begin{gathered}
k(t)=\int_{t-T}^{t} x_{0} e^{-\delta(t-\tau)} i(\tau) \mathrm{d} \tau \\
M(t)=\int_{t-T}^{t} \omega(t-\tau, x(\tau, t)) i(\tau) \mathrm{d} \tau=\int_{t-T}^{t}\left[\beta e^{\gamma(t-\tau)} x_{0}^{\mu} e^{-\delta \mu(t-\tau)}+\eta\right] i(\tau) \mathrm{d} \tau \\
=\int_{t-T}^{t}\left[\frac{\Omega}{\mu-1} e^{-\delta(t-\tau)}+\eta\right] i(\tau) \mathrm{d} \tau
\end{gathered}
$$

$$
i_{r}(t)=\delta k(t)+x_{0} e^{-\delta T} i(t-T)=x_{0}\left[\int_{t-T}^{t} \delta e^{-\delta(t-\tau)} i(\tau) \mathrm{d} \tau+e^{-\delta T} i(t-T)\right] .
$$

It should be also noted that by the same argument, we can also identify the optimal path of the endogenous obsolescence rate, $\xi(t)$, thanks to equation (8). We will be then able to investigate, among others, the issue of substitutability Vs complementarity of investment and maintenance expenditures. Because of the delayed integral representation of capital, maintenance and replacement investment in terms of the investment profile, see (10)-(12), the co-movements of each of the three variables and gross investment are not obvious at all. Yet (10)-(12) exhibit a quite striking property, at least for maintenance: by (11), past investment profile $i(z), t-T \leq z \leq t$, completely determines the size of maintenance expenditures at $t$. This is not at all the way maintenance expenditures are determined in the recent literature following the path-breaking contribution of McGrattan and Schmitz (1999). Therefore, our modeling does bring a new approach to the study of the relationship between investment and maintenance. 
Let us now dig deeper in our optimal control problem. If consumption is $c(t)=$ $y(t)-i(t)$, then the optimal control problem is:

subject to (for $t \geq 0)$ :

$$
\operatorname{Max} \int_{0}^{+\infty} e^{-\rho t} \frac{(y(t)-i(t))^{1-\sigma}}{1-\sigma} d t
$$

$$
y(t)=\int_{t-T}^{t}\left(\Omega e^{-\delta(t-s)}-\eta\right) i(s) d s .
$$

Note that in the expression above, for $s \in[-T, 0), i(s)$ is given and it is not part of the control, indeed it is the initial datum of the investment that we will denote by $\bar{\iota}(s) .{ }^{9}$ The integral equation (13) is the net production function obtained using (2) and (5), $T$ is the scrapping time identified by (6). It is possible to put the integral equation above into a more standard differential equation by differentiating it. Then we get the following state equation (for $t \geq 0$ ):

$$
\dot{y}(t)=(\Omega-\eta) i(t)-\delta \Omega \int_{-T}^{0} e^{\delta r} i(r+t) \mathrm{d} r, \quad \text { with } i(s)=\bar{\iota}(s) \text { when } s \in[-T, 0)
$$

The problem has some standard features but it entails a definitely non-standard characteristic. It has one control, investment, and one state, net output. The state equation is a delay-differential equation, which implies that the problem is infinite dimensional. This section is devoted to provide a closed-form solution to optimal dynamics, including asymptotic behavior. The technique used is inspired by Fabbri and Gozzi (2008a). We first explain its general principles, then we apply it to our model.

Apart from small changes some results given in this section can be proved as in Fabbri and Gozzi (2008a) so we do not repeat them here simply referring to that paper. Nevertheless some other results (especially Propositions 3.2, 3.3, 3.10, 3.11 and Theorems $3.2,3.3)$ are essentially different and cannot be derived from known results since they use the peculiar characteristics of our model. We prove them in the Appendix Appendix A.

\section{Solving the optimal control problem with dynamic programming}

The optimal control problem is treated following the procedure used by Fabbri and Gozzi (2008a). ${ }^{10}$ First we write the problem as an optimal control problem driven by a Delay Differential Equation (DDE) (Section 3.1) giving some preliminary results. Then we translate it $^{11}$ as an optimal control problem driven by an Ordinary Differential Equation (ODE) (without delay) in a suitable Hilbert space. At that point we apply the

\footnotetext{
${ }^{9}$ As mentioned above, the problem degenerates into a standard optimal control problem if the fixed cost $\eta$ goes to zero: in such a case, $T=\infty$ and (13) degenerates into a simple ordinary differential equation. We disregard this trivial case hereafter.

${ }^{10}$ We briefly outline the procedure in this subsection and we refer the reader to Fabbri and Gozzi (2008a) (Sections 1.2, 1.3, 2) and to its extended version Fabbri and Gozzi (2008b) for more details on the techniques and on the literature.

${ }^{11}$ Using the techniques introduced by Delfour (1986) and Vinter and Kwong (1981), see also Bensoussan et al. (2007) chapter 4.
} 
dynamic programming to this problem: we write and solve explicitly the HJB equation in the Hilbert space, we prove that the solution is the value function and we use the explicit expression of the value function to find the optimal feedback in closed form.

Solving the HJB equation is in general a difficult task: as well known, it is impossible in general to find an explicit solution even to finite dimensional HJB equations, so, $a$ fortiori, explicit solutions of infinite dimensional HJB equations are very rare. Here we have to use the particular structure of the problem. The explicit form of the value function allows us to solve the infinite dimensional problem and to find the closed loop solutions. All the job required to handle the infinite dimensional nature of the problem is performed in Subsection 3.2. Eventually we translate back the solution to the DDE setting and we find a closed loop solution of our original problem (Subsection 3.3).

\subsection{Statement of the optimal control problem in DDE form and preliminary results}

We first introduce a notation useful to rewrite more formally (14):

Notation 3.1 We call $\bar{\iota}:[-T, 0) \rightarrow \mathbb{R}^{+}$, the initial datum, $i:[0,+\infty) \rightarrow \mathbb{R}^{+}$the control strategy and $\tilde{\imath}:[-T,+\infty) \rightarrow \mathbb{R}^{+}$the function:

$$
\tilde{\imath}(s)=\left\{\begin{array}{lr}
\bar{\iota}(s) & s \in[-T, 0) \\
i(s) & s \in[0,+\infty) .
\end{array}\right.
$$

As explained before, standard pointwise initial conditions are not enough to determine solution paths to the DDEs involved. Rather we need an initial function on a particular time span depending on the particular delays involved. Accordingly, we shall work on functional spaces. Hereafter, we give the needed concepts to get through this problem, four useful functional spaces are defined.

$L^{2}([-T, 0) ; \mathbb{R})$ denotes to the space of all functions from $[-T, 0)$ to $\mathbb{R}$ that are Lebesgue measurable and square integrable. $L_{\text {loc }}^{2}([0,+\infty) ; \mathbb{R})$ is the space of all functions from $[0,+\infty)$ to $\mathbb{R}$ that are Lebesgue measurable and square integrable on all bounded intervals. $W^{1,2}([-T, 0) ; \mathbb{R})$ denotes the space of the functions in $L^{2}([-T, 0) ; \mathbb{R})$ whose first derivative exists and belongs to $L^{2}([-T, 0) ; \mathbb{R})$ too. Eventually $W_{\text {loc }}^{1,2}([0,+\infty) ; \mathbb{R})$ denotes the space of all functions belonging, together with their first derivatives, to $L_{\text {loc }}^{2}([0,+\infty) ; \mathbb{R})$. We denote by $L^{2}\left([-T, 0) ; \mathbb{R}^{+}\right)$the subset of $L^{2}([-T, 0) ; \mathbb{R})$ made by positive functions. Similarly we define $L_{\text {loc }}^{2}\left([0,+\infty) ; \mathbb{R}^{+}\right)$.

The state equation (14) can now be written as the following $\operatorname{DDE}\left(\right.$ on $\mathbb{R}^{+}$):

$$
\left\{\begin{array}{l}
\dot{y}(t)=(\Omega-\eta) \tilde{\imath}(t)-\delta \Omega \int_{-T}^{0} e^{\delta r} \tilde{\imath}(r+t) \mathrm{d} r \\
\tilde{\imath}(s)=\bar{\iota}(s) \forall s \in[-T, 0) \\
y(0)=\int_{-T}^{0} \bar{\iota}(s)\left(\Omega e^{\delta s}-\eta\right) \mathrm{d} s
\end{array}\right.
$$

where $\bar{\iota}(\cdot)$ and $y(0)$ are the initial conditions. ${ }^{12}$ We will assume $\bar{\iota}(\cdot) \geq 0$ and $\bar{\iota}(\cdot) \not \equiv 0$ so $y(0)>0$. Moreover we impose that $\bar{\iota}(\cdot) \in L^{2}\left([-T, 0), \mathbb{R}^{+}\right)$. Thanks to what we said in the previous sections $T, \Omega, \delta, \sigma, \rho$ are strictly positive constants, moreover we assume

\footnotetext{
${ }^{12}$ Indeed $y(0)$ is not a datum as it depends on $\bar{\iota}(\cdot)$ but (as we will see below) it is convenient to consider it a datum.
} 
that $\sigma \neq 1$. For every $i: \mathbb{R}^{+} \rightarrow \mathbb{R}$ locally integrable and every $\bar{\iota} \in L^{2}([-T, 0), \mathbb{R})$ there exists a unique locally absolutely continuous solution of (16) (that has to be understood in integral sense). Rewriting (13) using the notation introduced in (15) we have:

$$
y_{\bar{\iota}, i}(t)=\int_{t-T}^{t} \tilde{\imath}(s)\left(\Omega e^{-\delta(t-s)}-\eta\right) \mathrm{d} s .
$$

The functional to maximize is

over the set

$$
J(\bar{\iota} ; i) \stackrel{\text { def }}{=} \int_{0}^{\infty} e^{-\rho s} \frac{\left(y_{\bar{\iota}, i}(t)-i(t)\right)^{1-\sigma}}{(1-\sigma)} \mathrm{d} s
$$

$$
\mathcal{I}_{\bar{\iota}} \stackrel{\text { def }}{=}\left\{i(\cdot) \in L_{\text {loc }}^{2}\left([0,+\infty) ; \mathbb{R}^{+}\right): i(t) \in\left[0, y_{\bar{\iota}, i}(t)\right] \text { for almost all } t \in \mathbb{R}^{+}\right\} .
$$

The choice of $\mathcal{I}_{\bar{\iota}}$ ensures that $y_{\bar{\iota}, i}(\cdot) \in W_{\mathrm{loc}}^{1,2}\left([0,+\infty) ; \mathbb{R}^{+}\right)$. By the Sobolev embedding theorem this implies that $y_{\bar{\imath}, i}(\cdot)$ is continuous. We call Problem $(\boldsymbol{P})$ the problem of finding an optimal control strategy i.e. an $i^{*}(\cdot) \in \mathcal{I}_{\bar{\iota}}$ such that:

$$
J\left(\bar{\iota} ; i^{*}\right)=V(\bar{\iota}) \stackrel{\text { def }}{=} \sup _{i(\cdot) \in \mathcal{I}_{\bar{\iota}}}\left\{\int_{0}^{\infty} e^{-\rho s} \frac{\left(y_{\bar{\iota}, i}(t)-i(t)\right)^{1-\sigma}}{(1-\sigma)} \mathrm{d} s\right\} .
$$

$V$ called value function.

\subsubsection{Preliminary results}

We now give a preliminary study of the problem, in particular concerning the asymptotic behavior of admissible trajectories, the finiteness of the value function and the existence-and-uniqueness of optimal strategy.

Asymptotic behavior of admissible trajectories To find conditions ensuring the finiteness of the value function we need first to study the asymptotic behavior of the admissible trajectories, in particular to determine the maximum asymptotic growth rate of the output $y(\cdot)$. Equation (17) and the choice of $\mathcal{I}_{\bar{\iota}}$ suggest (since $\left(\Omega e^{\delta s}-\eta\right)$ is always positive in $[-T, 0])$ that the output is the highest possible one when $i(t)=y(t)$ for all $t \geq 0$. Setting $i(t)=y(t)$ in the state equation (16) we get the following DDE:

$$
\left\{\begin{array}{l}
\dot{y}(t)=(\Omega-\eta)(y(t))-\delta \Omega \int_{-T+t}^{t} e^{\delta r} y(r+t) \mathrm{d} r \\
y(t)=\int_{-T-t}^{0} \bar{l}(s+t)\left(\Omega e^{\delta s}-\eta\right) \mathrm{d} s \geq 0 \quad t \in[-T, 0] .
\end{array}\right.
$$

Such a DDE has a unique solution that we denote by $y_{M}(\cdot)$, the "candidate" maximal output. Proposition 3.1 will confirm such an intuition.

Proposition 3.1 Given an initial datum $\bar{\iota}(\cdot) \in L^{2}\left([-T, 0) ; \mathbb{R}^{+}\right)$and a control $i(\cdot) \in \mathcal{I}_{\bar{\iota}}$ we have that the solution $y_{\bar{\iota}, i}(\cdot)$ of $(16)$ is dominated at any time $t \geq 0$ by the solution $y_{M}(\cdot)$ of $(19)$.

Proof. See the proof of Proposition 2.1.3 of Fabbri and Gozzi (2008a).

Now we study the DDE (19). The associated characteristic equation is

$$
z=F_{1}(z):=\left\{\begin{array}{cl}
\Omega\left[1-e^{-\delta T}-\frac{\delta}{\delta+z}\left(1-e^{-(\delta+z) T}\right)\right] ; & \text { if } z \neq-\delta \\
\Omega\left[1-e^{-\delta T}-\delta T\right] ; & \text { if } z=-\delta
\end{array}\right.
$$


As shown in the subsequent proposition, the following hypothesis is crucial in studying the roots of the characteristic equation:

Hypothesis 3.1 We assume that $\frac{\Omega}{\delta}\left(1-e^{-\delta T}-\delta T e^{-\delta T}\right)>1$.

Hypothesis 3.1 is very interesting from the economic point of view. One easy way to get through it is to see its implications when the scrapping time is infinite (which happens when $\eta$ goes to zero) featuring a kind of standard AK limit case (with a constant saving rate equal to 1 ). In such a case, the hypothesis simply writes as $\Omega>\delta$, which is similar in spirit to the condition set in the standard AK model to ensure positive growth (that is $A>\delta$, where $\delta$ is physical depreciation). Actually $\Omega$ plays the role of parameter $A$ in the standard AK model: in our model with maintenance and endogenous utilization, the profitability of capital goods does not depend on parameter $A$ but also on those of the maintenance technology which matter crucially in the utilization decision. If the ratio $\frac{\Omega}{\delta}$ is not large enough, then even though all resources of the economy are channeled into investment, the process of capital accumulation need not be ever-lasting, and the economy need not grow in the long run. The following proposition identifies precisely this feature.

Proposition 3.2 There exists a strictly positive root $\pi$ to the characteristic equation (20) if and only if Hypothesis 3.1 is satisfied; in this case we have that:

(i) the only real roots of (20) are 0 and $\pi$ and they are simple;

(ii) all the complex roots are simple (except at most two).

Proof. See Appendix Appendix A.

From now on, we assume that Hypothesis 3.1 holds and we call $\pi$ the strictly positive root of the characteristic equation. As one can infer from the discussion above, $\pi$ is the maximal long-run growth rate the economy can generate. If Hypothesis 3.1 does not hold, $\pi$ cannot be positive. The next proposition formalizes this point in more accurate terms.

Proposition 3.3 Given any initial datum $\bar{\iota}(\cdot) \in L^{2}\left([-T, 0) ; \mathbb{R}^{+}\right)$the solution of (19) is continuous on $[0,+\infty)$ and

$$
y_{M}(t)=\alpha e^{\pi t}+o\left(e^{\pi t}\right) \quad \text { for } t \rightarrow+\infty
$$

where $\alpha$ is a coefficient depending on $\bar{\iota}$.

Proof. See Appendix Appendix A.

Finiteness of the value function As stated in the subsequent proposition, an immediate consequence of Proposition 3.1 and Proposition 3.3 is that the following hypothesis is sufficient to ensure that $V$ is finite:

Hypothesis 3.2 We assume that $\rho>\pi(1-\sigma)$.

Proposition 3.4 If Hypotheses 3.2 hold then $-\infty<V(\bar{\iota})<+\infty$ for all $\bar{\iota}$ in $L^{2}\left([-T, 0) ; \mathbb{R}^{+}\right)$.

Proof. See the proof of Propositions 2.1.10 and 2.1.11 of Fabbri and Gozzi (2008a). 
From now on we always assume that Hypotheses 3.1 and 3.2 are satisfied.

Existence and uniqueness of the optimal strategy The following proposition gives an existence and uniqueness result for the optimal strategy:

Proposition 3.5 Given an initial datum $\bar{\iota}(\cdot) \in L^{2}\left([-T, 0) ; \mathbb{R}^{+}\right)$there exists a unique optimal control in $\mathcal{I}_{\bar{\iota}}$, i.e. we can find in $\mathcal{I}_{\bar{\iota}}$ a unique admissible strategy $i^{*}(\cdot)$ such that $V(\bar{\iota})=J\left(\bar{\iota} ; i^{*}\right)$.

Proof. For the existence see the proof of Proposition 2.1.12 of Fabbri and Gozzi (2008a), for uniqueness it is enough to use in a standard way the strict concavity of the functional $J$.

The above existence result is an application of the direct method (i.e. we consider a maximizing sequence and we prove we can extract a subsequence converging to the optimal control) in the space of the Lebesgue measurable functions from $[0+\infty)$ to $\mathbb{R}$ integrable with respect to the measure $\mathrm{d} \mu(t)=e^{(\varepsilon-\pi) t} \mathrm{~d} t$ where $\mathrm{d} t$ is the Lebesgue measure and $\varepsilon$ a strictly positive constant (the same argument is used in Askenazy and Le Van (1999)).

\subsection{The equivalent problem in infinite dimension and its solution}

\subsubsection{Rewriting the problem in infinite dimension}

To rewrite our control problem with delay as a control problem without delay in infinite dimension the main difficulty is to define the new state variable (which will be called "structural state") and to find the new state equation that it satisfies. To accomplish this task we first write the DDE (16) in a more formal way, defining suitable functions and operators.

Given $t \geq 0$, the "history" of investments at time $t$ will be denoted by $\tilde{\imath}_{t}$ defined as:

$$
\tilde{\imath}_{t}:[-T, 0] \rightarrow \mathbb{R} ; \quad \tilde{\imath}_{t}(s)=\tilde{\imath}(t+s) .
$$

Moreover if we define the continuous linear mappings:

$$
\begin{gathered}
\mathcal{C}: C[-T, 0] \rightarrow \mathbb{R} ; \quad \mathcal{C}: f \mapsto(\Omega-\eta) f(0)-\delta \Omega \int_{-T}^{0} e^{\delta r} f(r) \mathrm{d} r \\
R: L^{2}([-T, 0), \mathbb{R}) \rightarrow \mathbb{R} ; \quad R: f \mapsto \int_{-T}^{0} f(s)\left(\Omega e^{\delta s}-\eta\right) \mathrm{d} s
\end{gathered}
$$

we can rewrite the state equation $(16)$ as $^{13}$

$$
\dot{y}(t)=\mathcal{C}\left(\tilde{\imath}_{t}\right), \quad t>0 ; \quad\left(y(0), \tilde{\imath}_{0}\right)=(R(\bar{\iota}), \bar{\iota}) .
$$

In treating the infinite dimensional problem, we will consider the state equation with general initial condition, without any interdependence between the initial conditions $\bar{\imath}(\cdot)$ and $y(0)$ :

Its solution is

$$
\dot{y}(t)=\mathcal{C}\left(\tilde{\imath}_{t}\right), \quad t>0 ; \quad\left(y(0), \tilde{\imath}_{0}\right)=\left(y_{0}, \bar{\iota}\right) .
$$

\footnotetext{
${ }^{13}$ Expression (24) could be undefined for some $t \geq 0$. Indeed we only need its integral form: $y(\tau)=$ $y(0)+\int_{0}^{\tau} \mathcal{C}\left(\tilde{\imath}_{t}\right) \mathrm{d} t$.
} 


$$
y_{\left(y_{0}, \bar{\iota}\right), i}(t)=y_{0}-R(\bar{\iota})+\int_{-T}^{0} \tilde{\imath}_{t}(s)\left(\Omega e^{\delta s}-\eta\right) \mathrm{d} s .
$$

From equation (17) it follows that $y_{(R(\bar{\iota}), \bar{\iota}), i}(\cdot)=y_{\bar{\iota}, i}(\cdot)$.

Now we are ready to write our infinite dimensional problem.

First we introduce the infinite dimensional space where the new state variable will live, which the same as in Fabbri and Gozzi (2008a):

$$
M^{2} \stackrel{\text { def }}{=} \mathbb{R} \times L^{2}([-T, 0), \mathbb{R}) .
$$

A generic element $x \in M^{2}$ is denoted as a couple $\left(x^{0}, x^{1}\right)$. The scalar product in $M^{2}$ is the standard one i.e.:

$$
\left\langle\left(x^{0}, x^{1}\right),\left(z^{0}, z^{1}\right)\right\rangle_{M^{2}} \stackrel{\text { def }}{=} x^{0} z^{0}+\left\langle x^{1}, z^{1}\right\rangle_{L^{2}}, \quad \text { for all }\left(x^{0}, x^{1}\right),\left(z^{0}, z^{1}\right) \in M^{2} .
$$

Second we define the state of the infinite dimensional problem: the so called "structural state". We denote by $F$ the function

$$
\begin{array}{ll}
F: L^{2}([-T, 0), \mathbb{R}) \rightarrow L^{2}([-T, 0), \mathbb{R}) ; & F: i \mapsto F(i) . \\
F(i)(s) \stackrel{\text { def }}{=} \int_{-T}^{s}-\delta \Omega i(-s+r) e^{\delta r} \mathrm{~d} r, & s \in[-T, 0) .
\end{array}
$$

Definition 3.1 Let $y_{0} \in \mathbb{R}^{+}, \bar{\iota}(\cdot) \in L^{2}\left([-T, 0), \mathbb{R}^{+}\right)$be the initial data; let $i(\cdot) \in$ $L_{\text {loc }}^{2}\left([0,+\infty) ; \mathbb{R}^{+}\right)$and $y_{\left(y_{0}, \bar{\iota}\right), i}(\cdot)$ as in $(26)$. Set $z \stackrel{\text { def }}{=}\left(y_{0}, F(\bar{\iota})\right) \in M^{2}$ (the initial datum in the Hilbert setting). The structural state of the system is the couple $x_{z, i}(t)=\left(x_{z, i}^{0}(t), x_{z, i}^{1}(t)\right) \stackrel{\text { def }}{=}\left(y_{\left(y_{0}, \bar{l}\right), i}(t), F\left(\tilde{\imath}_{t}\right)\right) \in M^{2}$ for all $t \geq 0$.

Third we write the equation satisfied by the new state variable. To do this we introduce the operator $A$ on $M^{2}$ :

$$
\left\{\begin{array}{l}
D(A) \stackrel{\text { def }}{=}\left\{\left(\psi^{0}, \psi^{1}\right) \in M^{2}: \psi^{1} \in W^{1,2}([-T, 0), \mathbb{R}), \psi^{0}=\psi^{1}(0)\right\} \\
A: D(A) \rightarrow M^{2} ; \quad A\left(\psi^{0}, \psi^{1}\right) \stackrel{\text { def }}{=}\left(0, D \psi^{1}\right)
\end{array}\right.
$$

With an abuse of notation we can identify, on $D(A), \psi^{1}(0)$ with $\psi^{0}$. So we can redefine the operator $\mathcal{C}$ on $D(A)$ as

$$
\left\{\begin{array}{l}
\mathcal{C}: D(A) \rightarrow \mathbb{R} \\
\mathcal{C}\left(\psi^{0}, \psi^{1}\right)=\mathcal{C} \psi^{1}=(\Omega-\eta) \psi^{1}(0)-\delta \Omega \int_{-T}^{0} e^{\delta r} \psi^{1}(r) \mathrm{d} r .
\end{array}\right.
$$

The theorem below links the dynamics of $y(\cdot)$ with that of the structural state.

Theorem 3.1 For all $\tau>0$, the structural state $x_{z, i}(\cdot)$ is the unique solution ${ }^{14}$ in

\footnotetext{
${ }^{14}$ Here the solution is meant in the following weak sense: for every $\psi \in D(A)$$$
\left\{\begin{array}{l}
\frac{d}{d t}\langle\psi, x(t)\rangle=\langle A \psi, x(t)\rangle_{M^{2}}+i(t) \mathcal{C}(\psi), \quad t \in(0, \tau] \\
\langle\psi, x(0)\rangle_{M^{2}}=\psi^{0} y_{0}+\left\langle\psi^{1}, F(\bar{\iota})\right\rangle_{L^{2}}
\end{array}\right.
$$ 


$$
\Pi \stackrel{\text { def }}{=}\left\{f \in C\left([0, \tau] ; M^{2}\right): \frac{d}{\mathrm{~d} t} f \in L^{2}\left((0, \tau) ; D(A)^{\prime}\right)\right\}
$$

of the equation:

$$
\left\{\begin{array}{l}
\frac{d}{\mathrm{~d} t} x(t)=A^{*} x(t)+\mathcal{C}^{*} i(t), \quad t>0 \\
x(0)=z=\left(y_{0}, F(\bar{\iota})\right)
\end{array}\right.
$$

where $A^{*}$ and $\mathcal{C}^{*}$ are the duals of the continuous linear operators $A: D(A) \rightarrow M^{2}$ and $\mathcal{C}: D(A) \rightarrow \mathbb{R}$.

Proof. See e.g. Bensoussan et al. (2007) Theorem 5.1 page 282.

Before proceeding, we need an existence and uniqueness result for the state equation for all initial conditions, not only for the ones given in (30).

Theorem 3.2 The equation

$$
\frac{d}{\mathrm{~d} t} x(t)=A^{*} x(t)+\mathcal{C}^{*} i(t), \quad t>0 ; \quad x(0)=z
$$

for $z \in M^{2}, i(\cdot) \in L_{\text {loc }}^{2}([0,+\infty) ; \mathbb{R})$ has a unique solution in $\Pi$ (defined in (29))

Proof. See Appendix Appendix A.

Fourth we formulate our optimal control problem in infinite dimension. The state space is $M^{2}$, the control space is $\mathbb{R}$, the time is continuous. The state equation in $M^{2}$ is given by

$$
\frac{d}{\mathrm{~d} t} x(t)=A^{*} x(t)+\mathcal{C}^{*} i(t) ; \quad t>0, \quad x(0)=z
$$

for $z \in M^{2}, i(\cdot) \in L_{\text {loc }}^{2}([0,+\infty) ; \mathbb{R})$. Thanks to Theorem 3.2, equation (31) has a unique solution $x_{z, i}(\cdot)$ in $\Pi$ (it extends the structural state defined in Definition 3.1 only for positive initial data and control), so $t \mapsto x_{z, i}^{0}(t)$ is continuous and it makes sense to consider the set of controls

$$
\mathcal{I}_{z}^{0} \stackrel{\text { def }}{=}\left\{i(\cdot) \in L_{\text {loc }}^{2}\left([0,+\infty) ; \mathbb{R}^{+}\right): i(t) \in\left[0, x_{z, i}^{0}(t)\right] \text { for a.e. } t \in \mathbb{R}^{+}\right\}
$$

The objective functional is

$$
J_{0}(z ; i) \stackrel{\text { def }}{=} \int_{0}^{\infty} e^{-\rho s} \frac{\left(x_{z, i}^{0}(t)-i(t)\right)^{1-\sigma}}{(1-\sigma)} \mathrm{d} s .
$$

The value function is then

$$
V_{0}(z) \stackrel{\text { def }}{=} \sup _{i(\cdot) \in \mathcal{I}_{z}} J_{0}(z ; i) \quad \text { if } \quad \mathcal{I}_{z} \neq \emptyset
$$

while $V_{0}(z) \stackrel{\text { def }}{=}-\infty$ if $\mathcal{I}_{z}^{0}=\emptyset$.

Remark 3.1 (Connection with the starting problem) If we have, for some $\bar{\iota}(\cdot) \in$ $L^{2}\left([-T, 0) ; \mathbb{R}^{+}\right), \quad z=(R(\bar{\iota}), F(\bar{\iota}))$, we find $\mathcal{I}_{z}^{0}=\mathcal{I}_{\bar{\iota}}, J_{0}(z ; i)=J(\bar{\iota} ; i)$ and $V_{0}(z)=V(\bar{\iota})$ and the solution of (31) is given by Theorem 3.1. 
3.2.2. Solving the infinite dimensional problem with dynamic programming

The HJB equation and its explicit solution The current value Hamiltonian is a function with values in the extended real line $\overline{\mathbb{R}}$ and defined on

$$
E \stackrel{\text { def }}{=}\left\{(x, P, i) \in M^{2} \times M^{2} \times \mathbb{R}: x^{0}>0, i \in\left[0, x^{0}\right], P \in D(A)\right\} .
$$

Its form is the following $\left(\langle i, \mathcal{C} P\rangle_{\mathbb{R}}\right.$ is the product on $\left.\mathbb{R}\right)$ :

$$
\mathcal{H}_{C V}\left(\left(x^{0}, x^{1}\right), P, i\right) \stackrel{\text { def }}{=}\left\langle\left(x^{0}, x^{1}\right), A P\right\rangle_{M^{2}}+\langle i, \mathcal{C} P\rangle_{\mathbb{R}}+\frac{\left(x^{0}-i\right)^{1-\sigma}}{(1-\sigma)}
$$

in the points in which $x^{0} \neq i$ and, for $\sigma \in(0,1)$, also for $x^{0}=i$. When $\sigma>1$ the above is not defined in the points in which $x^{0}=i$. In such points we set then $\mathcal{H}_{C V}=-\infty$.

The maximum value Hamiltonian (or simply Hamiltonian) is the real function defined on $G \stackrel{\text { def }}{=}\left\{(x, P) \in M^{2} \times M^{2}: x^{0}>0, P \in D(A)\right\}$ as

$$
\mathcal{H}(x, P)=\sup _{i \in\left[0, x^{0}\right]} \mathcal{H}_{C V}(x, P, i) .
$$

The HJB equation of our infinite dimensional control problem is then

$$
\rho V_{0}(x)-\mathcal{H}\left(x, D V_{0}(x)\right)=0 .
$$

We now recall the definition of solution of the HJB equation, and then we provide the explicit solution.

Definition 3.2 Let $\Theta$ be an open set of $M^{2}$ and $\Theta_{1} \subseteq \Theta$ a closed subset. A function $g \in$ $C^{1}(\Theta ; \mathbb{R})$ satisfies the HJB equation (32) on $\Theta_{1}$ if, for all $z \in \Theta_{1}$, we have $(x, D g(z)) \in G$ and

$$
\rho g(z)-\mathcal{H}(z, D g(z))=0 .
$$

Remark 3.2 If $P \in D(A)$ and $(\mathcal{C} P)^{-1 / \sigma} \in\left(0, x^{0}\right]$, by elementary arguments, the function $\mathcal{H}_{C V}(x, P, \cdot):\left[0, x^{0}\right] \rightarrow \mathbb{R}$ admits a unique maximum point given by

$$
i^{M A X}=x^{0}-(\mathcal{C} P)^{-1 / \sigma} \in\left[0, x^{0}\right)
$$

and then we can write the Hamiltonian in a simplified form:

$$
\mathcal{H}(x, P)=\langle x, A P\rangle_{M^{2}}+x^{0} \mathcal{C} P+\frac{\sigma}{1-\sigma}(\mathcal{C} P)^{\frac{\sigma-1}{\sigma}} .
$$

The expression for $i^{M A X}$ will be used to write the solution of the problem $\mathbf{P}$ in closed-loop form.

We define

$$
X \stackrel{\text { def }}{=}\left\{x \in M^{2}: x^{0}>0,\left(x^{0}+\int_{-T}^{0} e^{\pi s} x^{1}(s) \mathrm{d} s\right)>0\right\}
$$

and, calling $\nu \stackrel{\text { def }}{=} \frac{\rho-\pi(1-\sigma)}{\sigma \pi}$,

$$
Y \stackrel{\text { def }}{=}\left\{\left(x^{0}, x^{1}\right) \in X: \int_{-T}^{0} e^{\pi s} x^{1}(s) \mathrm{d} s \leq x^{0} \frac{1-\nu}{\nu}\right\} .
$$


It is easy to see that $X$ is an open set of $M^{2}$ and $Y \subseteq X$ is closed in $X$. We define, for $x \in M^{2}$, the quantity

$$
\Gamma_{0}(x) \stackrel{\text { def }}{=} x^{0}+\int_{-T}^{0} e^{\pi s} x^{1}(s) \mathrm{d} s .
$$

It is now possible to identify an explicit solution to the HJB equation (32) using the functional $\Gamma_{0}(\cdot)$ just defined. This is given in the next proposition.

Proposition 3.6 Under the Hypotheses 3.1 and 3.2 the function

$$
v: X \rightarrow \mathbb{R} ; \quad v(x) \stackrel{\text { def }}{=} a \Gamma_{0}(x)^{1-\sigma}
$$

with

$$
a \stackrel{\text { def }}{=} \nu^{-\sigma} \frac{1}{(1-\sigma) \pi}=\left(\frac{\rho-\pi(1-\sigma)}{\sigma \pi}\right)^{-\sigma} \frac{1}{(1-\sigma) \pi}
$$

is differentiable at all $x \in X$ and is a solution to the HJB equation (32) at all points of $Y$ in the sense of Definition 3.2.

Proof. See the proof of Proposition 2.2.9 of Fabbri and Gozzi (2008a).

The crucial proposition above deserves some comments. In the standard AK optimal growth model ${ }^{15}$, it is trivial to show that the solution to the corresponding HJB equation is actually $k^{1-\sigma}$ (possibly multiplied by a constant). In our infinite dimensional case, the role of capital is played by $\Gamma_{0}(x)$, which is the equivalent concept of capital in our infinite dimensional problem. This concept is introduced and justified clearly in Fabbri and Gozzi (2008a). ${ }^{16}$ Things will be immediately clear in Section 3.4 once we apply this methodology to our economic problem, that is once $\Gamma_{0}(x)$ is explicitly expressed in terms of the economic variables.

The Closed Loop control in infinite dimension Once the value function is identified, it is possible to study the closed loop control (feedback strategy), in other words, the policy function. Things are more complicated, but similar, in infinite dimension. Indeed, a preliminary definition is needed.

Definition 3.3 Given $\bar{\iota}(\cdot) \in L^{2}\left([-T, 0) ; \mathbb{R}^{+}\right)$with and $\bar{\iota} \not \equiv 0$, we call $\phi \in C\left(M^{2}\right)$ an admissible feedback strategy associated to $\bar{\iota}(\cdot)$ if the equation.

$$
\left\{\begin{array}{l}
\frac{d}{\mathrm{~d} t} x_{\phi}(t)=A^{*} x_{\phi}(t)+\mathcal{C}^{*}\left(\phi\left(x_{\phi}(t)\right)\right), t>0 \\
x_{\phi}(0)=(R(\bar{\iota}), F(\bar{\iota}))
\end{array}\right.
$$

has an unique solution $x_{\phi}(\cdot)$ in $\Pi$ and $\phi\left(x_{\phi}(\cdot)\right) \in \mathcal{I}_{\bar{L}}$. We denote the set of admissible feedback strategies associated to $\bar{\iota}(\cdot)$ with $A F S_{\bar{\iota}}$. We say that an admissible feedback strategy associated to $\bar{\iota}(\cdot)$ is an optimal feedback strategy associated to $\bar{\iota}(\cdot)$ if

$$
V(\bar{\iota})=V_{0}(R(\bar{\iota}), F(\bar{\iota}))=\int_{0}^{+\infty} e^{-\rho t} \frac{\left(x_{\phi}^{0}(t)-\phi\left(x_{\phi}(t)\right)\right)^{1-\sigma}}{(1-\sigma)} \mathrm{d} t .
$$

The set of optimal feedback strategies related to $\bar{\iota}(\cdot)$ will be denoted by $O F S_{\bar{\iota}}$.

\footnotetext{
${ }^{15}$ To be precise, without irreversibility constraint on investment.

${ }^{16}$ In particular, these authors show that when $T$ goes to infinity, featuring the standard AK model, their concept of equivalent capital converges well to the standard concept of capital in the one-dimensional case.
} 
We want to use the solution $v$ of the HJB equation (32) given in Proposition 3.6 to find the feedback strategy. $v$ is a solution of the HJB equation only in a part of the state space (the set $Y$ ). The function $v$ will be the value function and the associated closed loop strategy $\left(i^{M A X}\right.$ defined in Remark 3.2 where $P$ is the gradient of $v$ ) is optimal if and only if the related trajectory remains in $Y$. To guarantee this we have to impose another condition on the parameters of the problem. It substantially requires to rule out corner solutions.

Hypothesis 3.3 We assume that $\nu\left(1-\frac{\delta}{\delta+\pi} e^{-(\delta+\pi) T}\right) \leq 1$.

From now on we will assume that Hypotheses 3.1, 3.2, 3.3 are satisfied. At this stage, a further (anticipated) point can be made on the latter assumption. While it is designed to rule out corner solution, it is consistent with a further requirement. Indeed, we will see in Theorem 3.4 that the optimal paths grows at most as $e^{g t}$ where $g=\frac{\pi-\rho}{\sigma}$. So the condition to have a strictly positive growth of the BGP is $g>0$ i.e. $\rho<\pi$. It is easy to see that the condition $g>0$ implies Hypothesis 3.3 so our assumptions include all cases of strictly positive growth and also cases with possibly negative growth.

It's time now to state our results on optimal feedback strategies. The following theorem is useful.

Theorem 3.3 For all $\bar{\iota}(\cdot) \in L^{2}\left([-T, 0) ; \mathbb{R}^{+}\right)$with $\bar{\iota}(\cdot) \not \equiv 0$ the function

is in $O F S_{\bar{\iota}}$.

$$
\phi: M^{2} \rightarrow \mathbb{R} ; \quad \phi(x) \stackrel{\text { def }}{=} x^{0}-\nu \Gamma_{0}(x)
$$

Proof. See Appendix Appendix A.

Finally, we get the explicit expression for the value function $V_{0}$ :

Corollary 3.1 Given any $\bar{\iota}(\cdot) \in L^{2}\left([-T, 0) ; \mathbb{R}^{+}\right)$and setting $z=(R(\bar{\iota}), F(\bar{\iota}))$ we have that $V(\bar{\iota})=V_{0}(z)=v(z)$ where $v$ is given in Proposition 3.6.

Proof. See the proof of Corollary 2.2.14 of Fabbri and Gozzi (2008a).

\subsection{Going back: solution to the original problem}

Now we use the results we found in the infinite dimensional setting to solve the original optimal control problem $\boldsymbol{P}$. The results of this subsection are immediate corollaries of those of the previous one so we do not prove them. First of all observe that, given any $f(\cdot) \in L^{2}\left([-T, 0), \mathbb{R}^{+}\right)$and writing $z=(R(f), F(f))$, the quantity $\Gamma_{0}(z)$ defined in $(35)$ becomes

$$
\Gamma(f) \stackrel{\text { def }}{=} \Gamma_{0}(R(f), F(f))=y(0)-\int_{-T}^{0} e^{\pi s} \int_{-T}^{s} \delta \Omega f(-s+r) e^{\delta r} \mathrm{~d} r \mathrm{~d} s .
$$

We will use such an expression both when $f$ is the initial datum $(f=\bar{\iota})$ and when $f$ is the history $f=\tilde{\imath}_{t}$ for some $t \geq 0$. From Corollary 3.1 we get the following:

Proposition 3.7 The explicit expression for the value function $V$ related to problem $\mathbf{P}$ is

$$
V(\bar{\iota})=a(\Gamma(\bar{\iota}))^{1-\sigma}=a\left(y(0)-\int_{-T}^{0} e^{\pi s} \int_{-T}^{s} \delta \Omega \bar{\iota}(-s+r) e^{\delta r} \mathrm{~d} r \mathrm{~d} s\right)^{1-\sigma}
$$

where a is defined in (36). 
Using Theorem 3.3 we derive the optimal strategies of problem $\boldsymbol{P}$ in closed loop form:

Proposition 3.8 The optimal control $i^{*}$ for problem $\mathbf{P}$ and the related state trajectory $y^{*}$ satisfy, for all $t \geq 0$,

$$
i^{*}(t)=y^{*}(t)-\nu \Gamma\left(\tilde{i}_{t}^{*}(\cdot)\right) .
$$

In particular, for all $t \geq 0$, the optimal consumption path $c^{*}(t)=y^{*}(t)-i^{*}(t)$ satisfies $c^{*}(t)=\nu \Gamma\left(\tilde{\imath}_{t}^{*}(\cdot)\right)$

\subsection{Dynamic behavior of the optimal paths}

We analyze here the dynamic behavior of the optimal paths using the results of previous subsection. First we prove that the consumption $c^{*}(\cdot)$ is exponential and we write a suitable DDE for $y^{*}(\cdot)$ and $i^{*}(\cdot)$ :

Theorem 3.4 Let $\bar{\iota}(\cdot) \in L^{2}\left([-T, 0) ; \mathbb{R}^{+}\right)$. Taking the initial data $\left(y(0), \tilde{\imath}_{0}\right)=(R(\bar{\iota}), \bar{\iota})$ in (16), we have

where $g=\frac{\pi-\rho}{\sigma}$ and

$$
c^{*}(t)=\Lambda e^{g t}, \quad \forall t \geq 0
$$

$$
\Lambda \stackrel{\text { def }}{=} \nu \Gamma(\bar{\iota})=\nu\left(y(0)-\int_{-T}^{0} e^{\pi s} \int_{-T}^{s} \delta \Omega \bar{\iota}(-s+r) e^{\delta r} \mathrm{~d} r \mathrm{~d} s\right) .
$$

Moreover the optimal control $i^{*}(t)$ for problem $\mathbf{P}$ is the unique solution in $W_{\text {loc }}^{1,2}\left([0,+\infty) ; \mathbb{R}^{+}\right)$of the following DDE:

$$
i^{*}(t)=\int_{(t-T)}^{t} i^{*}(s)\left(\Omega e^{-\delta(t-s)}-\eta\right) \mathrm{d} s-\Lambda e^{g t}, \quad(t \geq 0) ;
$$

with the initial condition

$$
i_{0}^{*}(t)=\bar{\iota}(t), \quad t \in[-T, 0) .
$$

The optimal $y^{*}(\cdot)$ is the unique solution in $W_{\mathrm{loc}}^{1,2}\left([0,+\infty) ; \mathbb{R}^{+}\right)$of the following integral equation

$$
y^{*}(t)=\int_{(t-T) \wedge 0}^{0}\left(\Omega e^{\delta s}-\eta\right) \bar{\iota}(s) \mathrm{d} s+\int_{(t-T) \vee 0}^{t}\left[y^{*}(s)-\Lambda e^{g s}\right] \mathrm{d} s, \quad t \geq 0 .
$$

Proof. See the proof of Lemma 2.3.3 and Theorem 2.3.4 of Fabbri and Gozzi (2008a).

The previous proposition completely determines the optimal dynamics. Consistently with the AK vintage models studied in Boucekkine et al. (2005) and Fabbri and Gozzi (2008a), detrended consumption is constant as in the standard AK model. The growth rate $g=\frac{\pi-\rho}{\sigma}$ is easily interpretable having in mind the previous comments on $\pi$ in Section 3.2.1. The constant detrended consumption level, $\Lambda$, depends here on several factors. First of all, it depends on the whole investment history, and not only on the initial capital stock as in the standard AK model. ${ }^{17}$ Second, it depends on ALL the parameters of the model, including those of the maintenance technology through parameter $\Omega$. Countries having different maintenance strategies (reflected in different $\Omega$ ) are likely to have

\footnotetext{
${ }^{17}$ Countries sharing the same initial capital stock but having a different initial investment datum will have a different consumption level.
} 
different optimal consumption levels, and more importantly from the economic development point of view, different output levels. Indeed, once optimal consumption dynamics singled out, the dynamics of optimal investment (and thus output) follow simple linear (but non-autonomous) DDEs that can be solved either in closed form or numerically. Therefore in contrast to consumption, both investment and output optimal paths are not simple exponential functions. Before getting to the dynamics, let us investigate their asymptotic behavior. The following easy proposition is accurate enough.

Proposition 3.9 Defining, for $t \geq 0$, the optimal detrended paths as:

$$
y_{g}(t) \stackrel{\text { def }}{=} e^{-g t} y^{*}(t), \quad i_{g}(t) \stackrel{\text { def }}{=} e^{-g t} i^{*}(t), \quad c_{g}(t) \stackrel{\text { def }}{=} e^{-g t} c^{*}(t),
$$

we have that the optimal detrended consumption path $c_{g}(t)=\left(y_{g}(t)-i_{g}(t)\right)$ is constant and equal to $\Lambda$ (defined in (41)). Moreover there exist positive constants $i_{B}$ and $y_{B}$ such that

We have, when $g \neq-\delta$,

$$
\lim _{t \rightarrow+\infty} i_{g}(t)=i_{B} \quad \text { and } \quad \lim _{t \rightarrow+\infty} y_{g}(t)=y_{B} .
$$

$$
i_{B}=\frac{\Lambda}{\left(\int_{-T}^{0} \Omega\left(e^{\delta s}-e^{-\delta T}\right) e^{g s} \mathrm{~d} s\right)-1}, \quad y_{B}=\Lambda+\frac{\Lambda}{\left(\int_{-T}^{0} \Omega\left(e^{\delta s}-e^{-\delta T}\right) e^{g s} \mathrm{~d} s\right)-1} .
$$

Proof. See the proof of Proposition 2.3.5 of Fabbri and Gozzi (2008a).

Corollary 3.2 Define, for $t \geq 0$, the optimal detrended maintenance expenditure

$$
M_{g}(t) \stackrel{\text { def }}{=} e^{-g t} \int_{t-T}^{t}\left[\frac{\Omega}{\mu-1} e^{-\delta(t-\tau)}+\eta\right] i^{*}(\tau) \mathrm{d} \tau
$$

and the optimal detrended replacement investment

$$
i_{r_{g}}(t) \stackrel{\text { def }}{=} e^{-g t}\left(\frac{A}{\beta \mu}\right)^{\frac{1}{\mu-1}}\left[\int_{t-T}^{t} \delta e^{-\delta(t-\tau)} i^{*}(\tau) \mathrm{d} \tau+e^{-\delta T} i^{*}(t-T)\right] .
$$

There exist positive constants $M_{B}$ and $i_{r B}$ such that

$$
\lim _{t \rightarrow+\infty} M_{g}(t)=M_{B} \quad \text { and } \quad \lim _{t \rightarrow+\infty} i_{r_{g}}(t)=i_{r B}
$$

When $g \neq-\delta$,

$$
M_{B}=\int_{-T}^{0}\left(\frac{\Omega}{\mu-1} e^{\delta r}+\eta\right) i_{B} e^{g r} \mathrm{~d} r
$$

and

$$
i_{r B}=\left(\frac{A}{\beta \mu}\right)^{\frac{1}{\mu-1}} i_{B}\left[e^{-(\delta+g) T}+\int_{-T}^{0} \delta e^{\delta s} e^{g s} \mathrm{~d} s\right]
$$

Not surprisingly, both investment and output grow at the same asymptotic rate as consumption. More interestingly, the long-run investment and output levels $i_{B}$ and $y_{B}$ do depend on the initial conditions, which is a common feature of endogenous growth models, and on the relevant parameters of the model, including the parameters of the maintenance technology as expected. But the latter dependence is quite complicated to investigate analytically. We can develop a balanced growth path analysis as usual in growth theory, starting from the following definition. 
Definition 3.4 We will say that the system is on a Balanced Growth Path (BGP) if there exists $a_{0}, b_{0}>0$, and real numbers $a_{1}, b_{1}$ such that, for all $s \in[-T,+\infty)$, $\tilde{\imath}^{*}(s)=a_{0} e^{a_{1} s}$ and, for all $s \in[0,+\infty), y^{*}(s)=b_{0} e^{b_{1} s}$.

Notice that our definition accounts for the delayed nature of the operators driving the dynamics of the model like in equation (42). This features the dependence of the BGP on the initial investment datum. The next proposition highlights this sensitive property.

Proposition 3.10 If $g>0$ the system admits BGPs. More precisely, if $g>0$, all the possible $B G P s$ are those related to initial data of the form $\bar{\iota}(s)=a_{0} e^{g s}$ (for all $s \in[-T, 0))$ for some $a_{0} \in[0,+\infty)$. In this case we have that $\tilde{\imath}^{*}(s)=a_{0} e^{g s}$ for all $s \geq-T$ and $y^{*}(s)=b_{0} e^{g s}$ for all $s \geq 0$ where $b_{0}=\int_{-T}^{0} \Omega\left(e^{\delta s}-e^{-\delta T}\right) a_{0} e^{g s} \mathrm{~d} s$.

Proof. See Appendix Appendix A.

We study now the particular case in which the initial datum has the form $\bar{\iota}(s)=c e^{g_{0} s}$, for all $s \in[-T, 0)$, for some real constants $c$ and $g_{0}$ ( $c$ positive). This is the case we analyze in the numerical simulations in Section 4 and its simplicity allows to solve the integrals we found in our study and to provide a closed-form solution to the whole optimal dynamics, and not only the BGPs. The next proposition does the job.

Proposition 3.11 Let $c>0, g_{0}>0$ and let

$$
\bar{\iota}:[-T, 0] \rightarrow \mathbb{R} ; \quad \bar{\iota}(s)=c e^{g_{0} s} .
$$

Taking the initial data $\left(y(0), \tilde{\imath}_{0}\right)=(R(\bar{\iota}), \bar{\iota})$ in (16) we have:

(i) $\left\{\begin{array}{l}F(\bar{\iota}):[-T, 0) \rightarrow \mathbb{R} \\ F(\bar{\iota})(s)=-\delta \Omega c e^{-}\end{array}\right.$

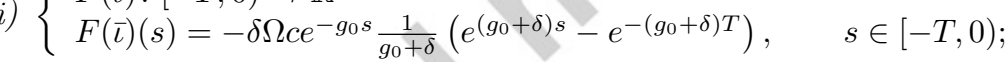

(ii) $\Gamma(\bar{\iota})=\left(\frac{\Omega c}{\delta+g_{0}}\left(1-e^{-\left(\delta+g_{0}\right) T}\right)-\frac{\eta c}{g_{0}}\left(1-e^{-g_{0} T}\right)-\frac{\delta \Omega c}{\delta+g_{0}} \frac{1}{\delta+\pi}\left(1-e^{-(\delta+\pi) T}\right)\right.$ $\left.+\frac{\delta \Omega c}{\delta+g_{0}} \frac{1}{\pi-g_{0}} e^{-\left(g_{0}+\delta\right) T}\left(1-e^{-\left(\pi-g_{0}\right) T}\right)\right) ;$

(iii) $\Lambda=\nu \Gamma(\bar{\iota})$ and $V(\bar{\iota})=\nu^{-\sigma} \frac{1}{(1-\sigma) \pi} \Gamma(\bar{\iota})^{1-\sigma}$;

(iv) $i_{B}=\frac{1}{\Omega\left(\frac{1}{g+\delta}\left(1-e^{-(\delta+g) T}\right)-\frac{e^{-\delta T}}{g}\left(1-e^{-g T}\right)\right)-1} \Lambda$ and $y_{B}=\left(i_{B}+1\right) \Lambda$.

Proof. See Appendix Appendix A.

Items (i) and (ii) of the proposition give the explicit forms of functionals $F($.$) and$ $\Gamma($.) (applied to initial datum) which are enough to obtain a closed form solution to optimal investment dynamics by Theorem 3.8 in Section 3.4. We shall now investigate the implications of such solutions for the optimal investment and maintenance co-movements. 


\section{Optimal investment and maintenance co-movements}

In this section, we run some numerical experiments using the analytical findings of the previous section. We choose to present the results of a non-anticipated, permanent and positive shock on the productivity parameters $A .{ }^{18}$ Before the shock, the parameters are chosen so that the long-run growth rate of the economy is between $2 \%$ and $3 \%$, the ratio maintenance to investment is about $50 \%$ as documented by McGrattan and Schmitz (1999), capital lifetime is between 10 and 15 years, and an obsolescence rate between $1 \%$ and $3 \%$, both figures being quite common in the literature. ${ }^{19}$ Figures 1 to 4 show an example of the optimal dynamics following a non-anticipated permanent $1 \%$ increase in the productivity parameter $A$ for investment, maintenance, replacement investment and the obsolescence rate. The first three variables are detrended in the sense that each variable, say $q(t)$, is reported as $\hat{q}(t)=q(t) e^{-g t}, \forall t \geq 0$, where $g$ is the long-run growth rate resulting from the permanent shock. Needless to say, the value of machines' lifetime, $T$, is adjusted from $t=0$, since the $A$-shock also affects this variable. ${ }^{20}$ Last by not least, the figures are generated with an initial investment profile of the form $\bar{\iota}(s)=c e^{g_{0} s}$ as anticipated in the previous section. In the numerical illustration considered here, we set $c=1$ and $g_{0}$ is the long-run growth rate of the economy before the $A$-shock, here $g_{0}=2.54 \%$.

Figures 1 and 2 give the optimal investment and maintenance dynamics respectively. Because initial investment profile is $\bar{\iota}(s)=e^{g_{0} s}$, detrended investment satisfies:

$$
\lim _{t \rightarrow 0^{-}} \hat{\bar{l}}(t)=\lim _{t \rightarrow 0^{-}} e^{\left(g_{0}-g\right) t}=1 .
$$

Figure 1 thus shows clearly that the permanent technological stimulus induces a neat increase in (detrended) investment just after the shock relative to the corresponding level just before the shock: Investment jumps at $t=0$ ! This discontinuity is quite common in the DDE literature (see Boucekkine et al., 2005). More importantly, Figure 1 captures very well the essential features of optimal adjustment in this kind of models: Investment is stimulated in the short and long-run, though the long-run "multiplier" is smaller. In the middle, the dynamics are much more persistent than in a model with homogeneous capital due to replacement dynamics. Due to these dynamics, which are themselves induced by the optimal constant lifetime of machines, convergence to the new balanced growth paths is oscillatory (although the scale of the figures does not reflect this so neatly after $t=\frac{3 T}{2}$ ). This is again consistent with the available AK theory with vintage capital. Figure 2 displays the optimal dynamics of capital maintenance. For a careful interpretation of the dynamics, the reader should note that given our parameterization

$$
\lim _{t \rightarrow 0^{-}} \hat{M}(t)=0.5008
$$

\footnotetext{
${ }^{18}$ We have also studied the consequences of shocks on the support cost, $\eta$.

${ }^{19}$ More precisely, three parameters were fixed and four varied. The fixed parameters are: $\beta=1, \sigma=4$ and $\rho=3 \%$. The four varying parameters were: $\gamma, A, \eta$ and $\mu$.

${ }^{20}$ In Figure 1 to 4 , we have: $A=0.62$ before the shock, $\gamma=1.89, \eta=0.0296$ and $\mu=10$. This implies a long-run growth rate equal to $2.54 \%$ before the shock, and equal to $2.68 \%$ after the shock. The ratio maintenance expenditures to investment is close to $50 \%$, and the machines' lifetime is equal to 12.5 before the shock. The latter increases very slightly to 12.55 years after the shock. The resulting obsolescence rate is equal to $1.30 \%$ before the shock.
} 


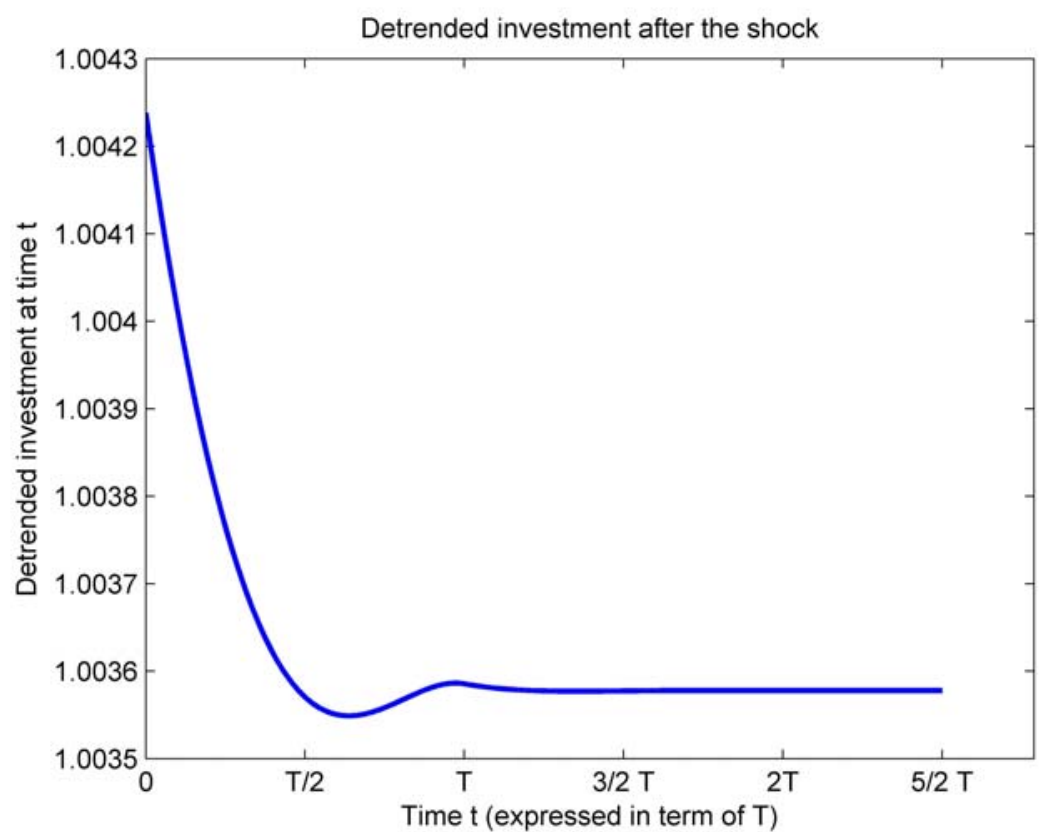

Figure 1: Optimal response of the detrended investment $i_{g}(t)$ (see (44))

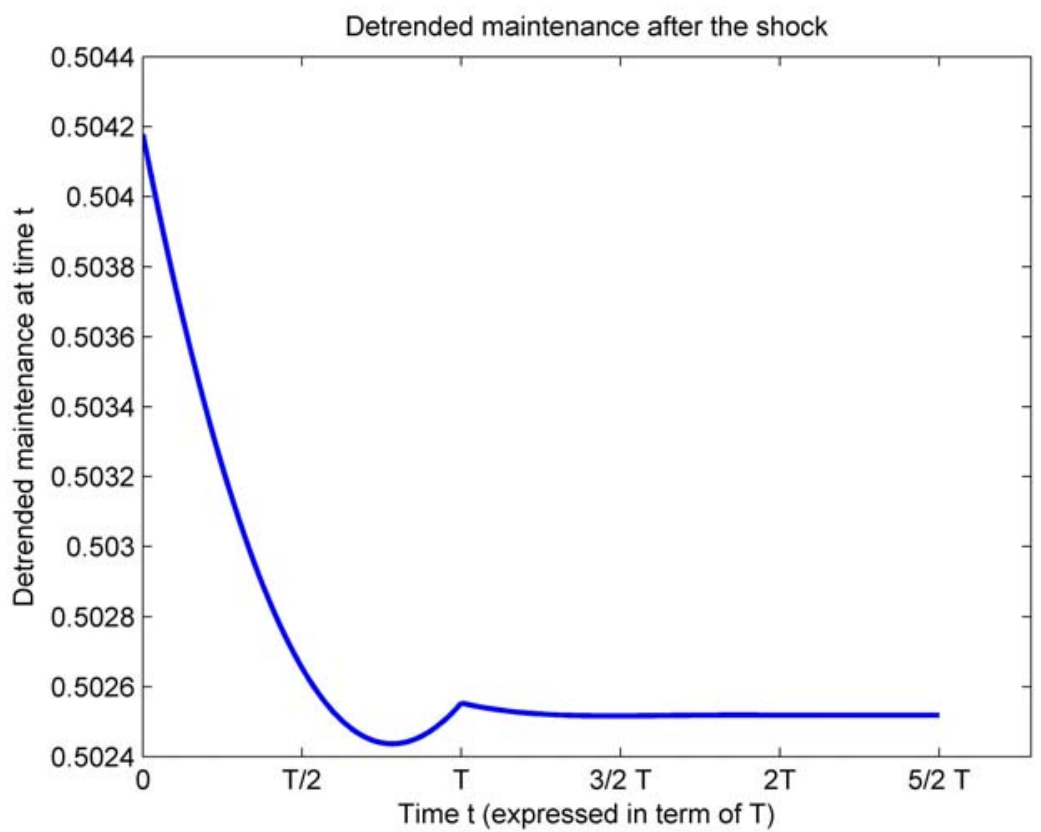

Figure 2: Optimal response of the detrended maintenance $M_{g}(t)$ (see (46)) 22 
Figure 2 thus implies even the maintenance variable jumps at $t=0$, which is much more surprising than the investment jump. Indeed, $M(t)$ is not a control variable, and moreover, it is a weighted time integral of the investment profile from $t$ to $t-T$, as reflected in equation (11). So why this jump? This jump occurs because the increase in the productivity parameter $A$ changes the value of optimal lifetime from $t=0$. Therefore the lower integration bound appearing in (11) gets modified, inducing the jump. More interestingly, one can notice that investment and maintenance move in the same way. Maintenance goes up at $t=0$ because capital lifetime increases (slightly) inducing a larger set of vintages to maintain. The remaining maintenance dynamics are explained by investment dynamics given the integral law of motion (11). ${ }^{21}$ Overall, one can see that optimal investment and maintenance patterns show a great degree of complementarity, a property which we found to be robust to a wide variety of shocks and initial conditions. ${ }^{22}$

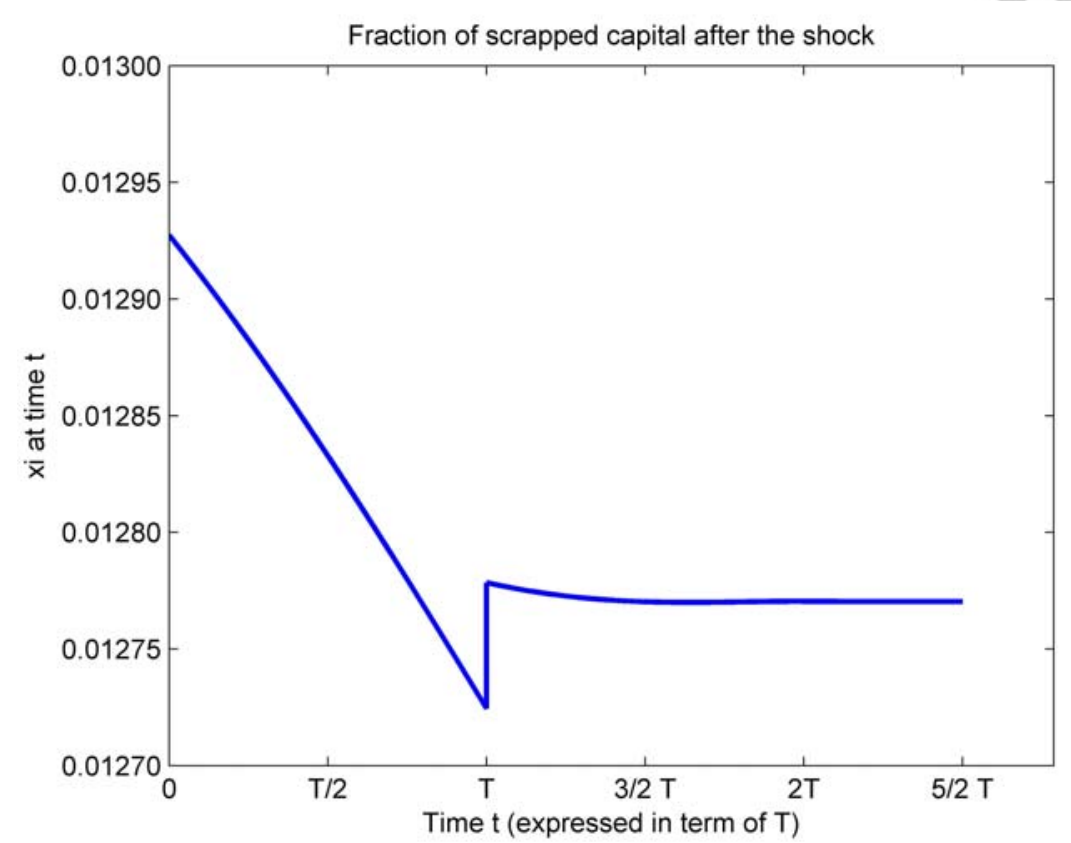

Figure 3: Behavior of the optimal obsolescence rate $\xi(t)$ (see (8)) along the optimal path

Figures 3 and 4 give the optimal dynamics of the obsolescence rate $\xi(t)$ and replacement investment. The former is given by equation (8), and the latter by (12). Two important characteristics have to be mentioned. First of all, and this may be the most salient feature of Figures 3 and 4, the two paths show a discontinuity at $t=T$, which comes trivially from the fact that investment is jumping at $t=0$. Indeed, both variables

\footnotetext{
${ }^{21}$ One could differentiate (11) with respect to time to make explicit the link.

${ }^{22}$ In particular, we try shocks on the parameters of the maintenance technology.
} 
depend on the term $i(t-T)$. Second, for the same reason as for maintenance expenditures, there is also a much less apparent jump of replacement investment and the rate of obsolescence at $t=0$. Instantaneous adjustment of $T$ to its new value, following the $A$-shock, do include jumps of the two variables at $t=0$. More precisely, both the rate of obsolescence and replacement investment experience a downward jump at $t=0 .{ }^{23}$ While investment shifts upward, replacement investment goes down just after the shock, which is a powerful reason to distinguish between the two components of investment in any business cycle study. Why? As the value of the optimal lifetime of machines goes up, less machines will be thrown out just after the shock, the obsolescence costs will be lower (which is reflected in the initial downward shift in the rate of obsolescence), and replacement investment too. The remaining dynamics derive from investment dynamics according to the lagged structures appearing in the law of motions (8) and (12), ultimately yielding oscillatory convergence to the new balanced growth path.

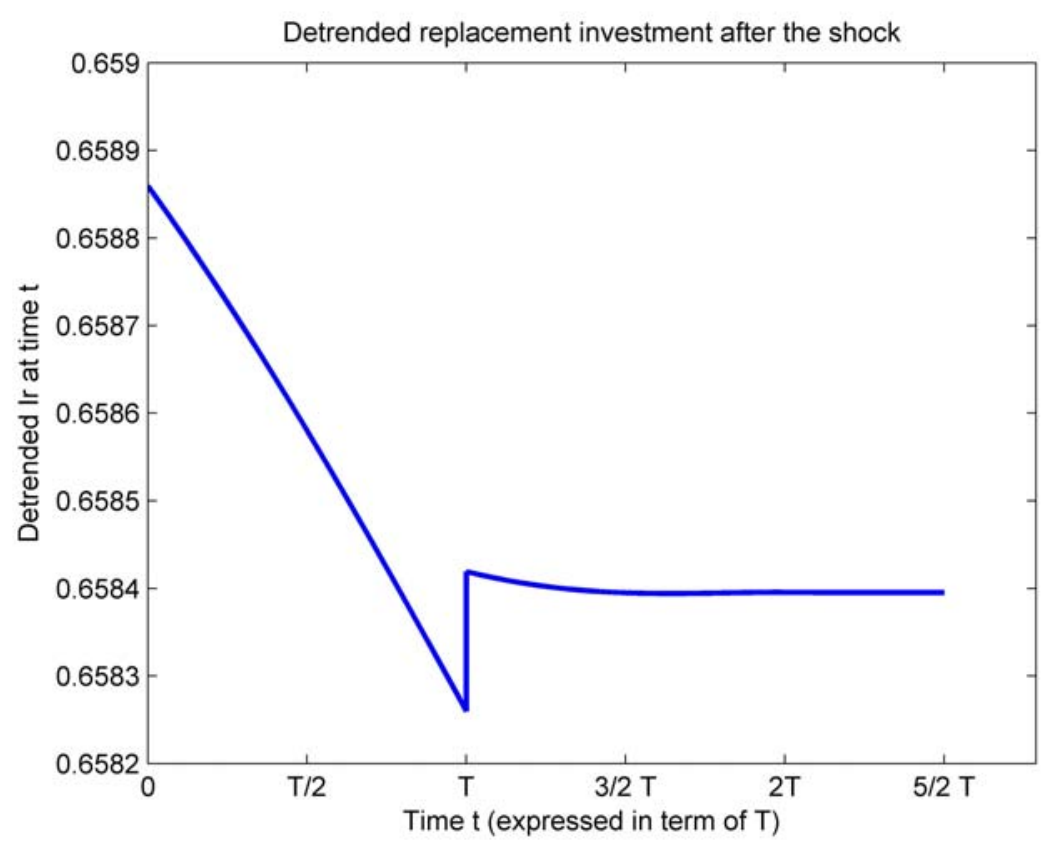

Figure 4: Optimal response of the detrended replacement investment $i_{r_{g}}(t)$ (see (47))

\section{Conclusions}

In this paper, we have considered a benchmark model of the AK type, incorporating maintenance costs and allowing for endogenous obsolescence, in order to study optimal

\footnotetext{
${ }^{23}$ To be more precise, the rate of obsolescence and replacement investment (detrended) are equal to $1.30 \%$ and 0.659 respectively just before the shock.
} 
investment and maintenance co-movements in a framework where such co-movements do no rely exclusively on a postulated $a d-h o c$ depreciation function as it is usually done in the related literature. At equilibrium, we prove that current optimal maintenance is a weighted integral of past investment profile with time span depending on the endogenous pace of obsolescence. Adapting a recent analytical approach to vintage models proposed by Fabbri and Gozzi (2008a), we have been able to find the optimal dynamics of the model in closed form. Completing the job with numerical assessment, we found that investment and maintenance move in the same way in response to neutral technological shocks, which suggest that they act as complementary to each other, which seems to be consistent with data.

Deviating from AK framework for more comprehensive empirical work will of course disable the analytical approach mostly taken in this paper. For example, with less stylized Cobb-Douglas technologies as in Boucekkine et al. (2009), the involved state equations are no longer linear delay differential equations, so that one can only resort to a full computational approach. Moreover, accounting for investment-specific technological progress will make the analysis of investment and maintenance co-movements even trickier. Indeed, while accelerations in neutral technical progress lengthen capital lifetime as shown in this paper, investment-specific technological progress tends rather to decrease it (see again Boucekkine et al. (2009)). In a model with both forms of technological progress, investment and maintenance co-movements would therefore depend a lot on the composition of technological progress and on the sensitivity of the scrapping decision to each form of technical progress. This goes much beyond the scope of this paper. But precisely because the latter general problem seems quite hard to address properly, we do believe that the benchmark analysis provided in this article is a necessary step in this research program.

\section{Appendix A. Appendix: Proofs}

We start by proving the following lemma that will be used in the proof of Proposition 3.2

Lemma Appendix A.1 The function $F_{1}$ defined in (20) is strictly increasing and strictly concave. Moreover $F_{1}(-\infty)=-\infty, F_{1}(0)=0, F_{1}(+\infty)=\Omega\left(1-e^{-\delta T}\right)$ and $F_{1}^{\prime}(0)=$ $\frac{\Omega}{\delta}\left(1-e^{-\delta T}-\delta T e^{-\delta T}\right)$.

Proof. It is not difficult to see that, setting $h(x)=1-e^{-x}-x e^{-x}$, we have

$$
F_{1}^{\prime}(z)=\frac{\Omega \delta}{(\delta+z)^{2}} h((\delta+z) T), \quad \text { if } \quad z \neq-\delta \quad \text { and } \quad F_{1}^{\prime}(z)=\frac{1}{2} \Omega \delta T^{2}, \quad \text { if } \quad z=-\delta
$$

while, setting $g(x)=-2 h(x)+x h^{\prime}(x)=-2+2 e^{-x}+2 x e^{-x}+x^{2} e^{-x}$ we get

$$
F_{1}^{\prime \prime}(z)=\frac{\Omega \delta}{(\delta+z)^{3}} g((\delta+z) T), \quad \text { if } \quad z \neq-\delta \quad \text { and } \quad F_{1}^{\prime \prime}(z)=-\frac{1}{3} \Omega \delta T^{3}, \quad \text { if } \quad z=-\delta .
$$

Now by simple computations we can see that

$$
h(x)>0, \quad \forall x \in \mathbb{R}-\{0\} \quad \text { and } \quad \frac{g(x)}{x^{3}}<0, \quad \forall x \in \mathbb{R}-\{0\} .
$$

so the whole claim of the Lemma easily follows. 


\section{Proof of Proposition 3.2}

The first statement is an immediate consequence of Lemma Appendix A.1. Statement (i) follows observing that $F_{1}^{\prime}(0)>1$ by Hypothesis 3.1 and $F_{1}^{\prime}(\pi)<1$ by the strict monotonicity and concavity of $F_{1}$.

To prove (ii) we first observe that (20) can be rewritten, multiplying by $\delta+z \neq 0$, as

$$
z^{2}+\left[-\Omega\left(1-e^{-\delta T}\right)+\delta\right] z+\delta \Omega e^{-\delta T}-\delta \Omega e^{-(\delta+z) T}=0 .
$$

All complex roots of (20) are also roots of (A.3): they are at most countable and have the form $\lambda_{k}=a_{k} \pm i b_{k}$ for two sequences of real numbers $\left\{a_{k}\right\}$ and $\left\{b_{k}\right\}$. Now a characteristic root is nonsimple if and only if it satisfies also the equation $2 z+\left(-\Omega\left(\left(1-e^{-\delta T}\right)+\delta\right)+\delta \Omega T e^{-(\delta+z) T}=0\right.$. Combining the two equations we get that a non simple characteristic root $z$ must solve the quadratic equation $z^{2}+\left[\frac{2}{T}-\Omega\left(1-e^{-\delta T}\right)+\delta\right] z+\delta \Omega e^{-\delta T}+\frac{\Omega\left(\left(1-e^{-\delta T}\right)-\delta\right.}{T}$. This means that there are at most two complex conjugate non simple characteristic roots.

\section{Proof of Proposition 3.3}

By Diekmann et al. (1995) page. 34 and the Proposition 3.2 it follows that the solution of (19) is continuous on $\mathbb{R}^{+}$and

$$
y_{M}(t)=o\left(e^{\pi t}\right)+\alpha e^{\pi t}+\sum_{j=1}^{N} p_{j}(t) e^{\lambda_{j} t} \quad \text { for } t \rightarrow+\infty
$$

where $\lambda_{j}$ are the finitely many roots of the characteristic equation with real part exceeding $\pi$ and $p^{j}$ are $\mathbb{C}$-valued polynomial in $t$. Now we can easily see that the part due to the trigonometric polynomial $\sum_{j=1}^{N} p_{j}(t) e^{\lambda_{j} t}$ vanish because the solution remain always positive, due to the following property: if the initial datum $\bar{\iota}(\cdot)$ is not identically zero then $y_{M}(t)$ remains positive for all $t$. Indeed, the solution is continuous and its value in 0 is strictly positive. If there exists a first point $\bar{t}$ in which the solution is zero it satisfies:

$$
y_{M}(\bar{t})=\int_{(\bar{t}-T) \wedge 0}^{\bar{t}} y_{M}(s+t)\left(\Omega e^{\delta s}-\eta\right) \mathrm{d} s+\int_{(\bar{t}-T) \vee 0}^{0} \bar{l}(s+t)\left(\Omega e^{\delta s}-\eta\right) \mathrm{d} s
$$

but the right hand side of equation is strictly positive because $\bar{t}$ is the first positive point in which $y_{M}(t)=0$.

Proof of Theorem 3.2

Thanks to Theorem 5.1 page 282 in Bensoussan et al. (2007) we know that a solution exists for all $i(\cdot) \in L_{\text {loc }}^{2}([0,+\infty) ; \mathbb{R})$ when the initial datum $z$ is in $N:=\mathbb{R} \times F\left(L^{2}([-T, 0) ; \mathbb{R})\right)$. Moreover the same theorem ensures that having a weak solution as defined in $(28)$ is equivalent to having a solution in the following mild sense: $x(\cdot)$ in $\Pi$ is a (mild) solution if, for all $\psi \in D(A)$,

$$
\langle\psi, x(t)\rangle=\left\langle e^{t A} \psi, z\right\rangle+\int_{0}^{t} \mathcal{C}\left(e^{(t-r) A} \psi\right) i(s) \mathrm{d} s
$$

that gives, for all $z_{1}, z_{2} \in N$ and $i(\cdot) \in L_{\text {loc }}^{2}([0,+\infty) ; \mathbb{R}),\left(x_{z_{1}, i}-x_{z_{2}, i}\right)=e^{t A^{*}}\left(z_{1}-z_{2}\right)$. Moreover it is easy to see that $N$ is dense in $M^{2}$ and, fixed $i(\cdot) \in L_{\text {loc }}^{2}([0,+\infty) ; \mathbb{R})$, given $z \in M^{2}$ and $z_{n} \in N$ with $z_{n} \underset{M^{2}}{\stackrel{n \rightarrow \infty}{\longrightarrow}} z$, that $x_{z_{n}, i}(\cdot)$ converges in $\Pi$ to a $x_{z, i}(\cdot)$ that is the solution we were looking for.

Proof of Theorem 3.3

First of all we prove that $\phi \in A F S_{\bar{\iota}}$. We divide the proof in two steps. Step 1: We claim that

$$
\left\{\begin{array}{l}
\frac{d}{\mathrm{~d} t} x_{\phi}(t)=A^{*} x_{\phi}(t)+\mathcal{C}^{*}\left(\phi\left(x_{\phi}(t)\right)\right), t>0 \\
x_{\phi}(0)=z=(R(\bar{\iota}), F(\bar{\iota}))
\end{array}\right.
$$


has a unique solution in $\Pi$.

To prove this first step we consider the solution $i(\cdot)$ of the following delay differential equation

$$
\left\{\begin{aligned}
i(t) & =(1-\nu)\left(\int_{(t-T)}^{t} i(s)\left(\Omega e^{-\delta(t-s)}-\eta\right) \mathrm{d} s\right)-\nu \int_{-T}^{0} e^{\pi s} F\left(i_{t}\right)(s) \mathrm{d} s \\
i(s) & =\bar{\iota} \forall s \in[-T, 0)
\end{aligned}\right.
$$

that has an absolute continuous solution $i$ on $[0,+\infty)$ (see for example Bensoussan et al. (2007) page 287 for a proof). Then we consider the equation

$$
\left\{\begin{array}{l}
\frac{d}{\mathrm{~d} t} x=A^{*} x+\mathcal{C}^{*}(i(t)), \quad t>0 \\
x(0)=z=(R(\bar{\iota}), F(\bar{\iota}))
\end{array}\right.
$$

We know, thanks to Theorem 3.1, that the only solution in $\Pi$ of $(\mathrm{A} .7)$ is $x(t):=\left(y(t), F\left(i_{t}\right)\right)$ for all $t \geq 0$, where $y(\cdot)$ is the solution of

$$
\dot{y}(t)=\mathcal{C}\left(i_{t}\right) ; \quad\left(y(0), i_{0}\right)=(R(\bar{\iota}), \bar{\iota}) .
$$

We claim that $x(t)$ is solution of (A.5) indeed

$$
\phi(x(t))=y(t)-\nu\left(\int_{-T}^{0} e^{\pi s} F\left(i_{t}\right)(s) \mathrm{d} s+y(t)\right)
$$

and so (by (A.6):

$$
\phi(x(t))=y(t)(1-\nu)+i(t)-(1-\nu)\left(\int_{(t-T)}^{t} i(s)\left(\Omega e^{-\delta(t-s)}-\eta\right) \mathrm{d} s\right)
$$

and by (A.7) we conclude that $\phi(x(t))=i(t)$ for all $t \geq 0$ and so $x(\cdot)=x_{\phi}(\cdot)$ is a solution of (A.5) and is in $\Pi$. Moreover thanks to the linearity of $\phi$ it is easy to observe that $x_{\phi}(\cdot)$ is the unique solution in $\Pi$.

Step 2: We claim that $\phi\left(x_{\phi}(\cdot)\right) \in \mathcal{I}_{\bar{\iota}}$.

We have to prove that, for all $t \geq 0, \phi\left(x_{\phi}(t)\right)=i(t) \in\left[0, x_{\phi}^{0}(t)\right]$. First we prove that $i(t) \geq 0$, i.e. that $\nu\left(\int_{-T}^{0} e^{\pi s} x_{\phi}^{1}(t)(s) \mathrm{d} s+x_{\phi}^{0}(t)\right) \leq x_{\phi}^{0}(t)$. By Step 1 we have $x_{\phi}(t)=\left(R\left(i_{t}\right), F\left(i_{t}\right)\right)$ so, using the definitions of $R$ and $F$ and the Fubini Theorem we get:

$$
\begin{gathered}
\nu\left(\int_{-T}^{0} e^{\pi s} x_{\phi}^{1}(t)(s) \mathrm{d} s+x_{\phi}^{0}(t)\right)=\nu\left(\int _ { - T } ^ { 0 } \Omega i _ { t } ( r ) \left(e^{\delta r}-e^{-\delta T}\right.\right. \\
\left.\left.-\frac{\delta}{\delta+\pi} e^{\delta r} e^{(\delta+\pi) r}+\frac{\delta}{\delta+\pi} e^{\delta r} e^{-(\delta+\pi) T}\right) \mathrm{~d} r\right) \\
\leq \nu\left(\int_{-T}^{0} \Omega i_{t}(r)\left(\left(e^{\delta r}-e^{-\delta T}\right)\left(1-\frac{\delta}{\delta+\pi} e^{-T(\delta+\pi)}\right)\right) \mathrm{d} r\right) \\
=\nu\left(1-\frac{\delta}{\delta+\pi} e^{-(\delta+\pi) T}\right) R\left(i_{t}\right) \leq R\left(i_{t}\right)=x_{\phi}^{0}(t)
\end{gathered}
$$

where the last inequality follows by Hypothesis 3.3 .

We prove now that, for all $t \geq 0, i(t) \leq x_{\phi}^{0}(t)$ i.e. that

$\nu\left(x_{\phi}^{0}(t)+\int_{-T}^{0} e^{\pi s} x_{\phi}^{1}(t)(s) \mathrm{d} s\right) \geq 0$. Using the expressions of $R$ and $F$ as above we get

$$
x_{\phi}^{0}(t)+\int_{-T}^{0} e^{\pi s} x_{\phi}^{1}(t)(s) \mathrm{d} s=R\left(i_{t}\right)+\int_{-T}^{0} e^{\pi s} F\left(i_{t}\right)(s) \mathrm{d} s=\int_{-T}^{0} \Omega i(r+t) \Phi(r) \mathrm{d} r
$$

where the function $\Phi:[-T, 0] \rightarrow \mathbb{R}$ is given by

$$
\Phi: r \mapsto e^{\delta r}-e^{-\delta T}-\frac{\delta}{\delta+\pi} e^{\delta r}\left(e^{(\delta+\pi) r}-e^{-(\delta+\pi) T}\right) .
$$

Now by elementary computation we can prove that $\Phi(-T)=0$ and $\Phi(r)>0$ for all $r \in(-T, 0]$. Using that $i(t) \geq 0$ for all $t>-T$ we have the claim. 
This concludes the proof of the fact that $\phi$ is an admissible strategy related to $\bar{\iota}$. The optimality can be proved using the same arguments used by Fabbri and Gozzi (2008a) in the proof of Theorem II.2.13.

Proof of Proposition 3.10

It is enough to try the solution $a_{0} e^{a_{1} s}$ in (42). We find:

then

$$
\begin{gathered}
a_{0} e^{a_{1} s}=\left(\int_{-T}^{0} \Omega e^{\left(\delta+a_{1}\right) r} \mathrm{~d} r-\int_{-T}^{0} \Omega e^{a_{1} r} e^{-\delta T} \mathrm{~d} r\right) a_{0} e^{a_{1} s}\left(\frac{\pi-\rho}{\sigma \pi}\right) \\
+a_{0} e^{a_{1} s} \nu \int_{-T}^{0} e^{\pi \tau} \int_{-T}^{\tau} e^{-a_{1} \tau} \delta \Omega e^{\left(a_{1}+\delta\right) r} \mathrm{~d} r \mathrm{~d} \tau
\end{gathered}
$$

$$
\begin{aligned}
1=\Sigma\left(a_{1}\right) \stackrel{\text { def }}{=}\left(\Omega \frac{1}{\delta+a_{1}}\left(1-e^{-\left(\delta+a_{1}\right) T}\right)-\Omega e^{-\delta T} \frac{1}{a_{1}}\left(1-e^{-a_{1} T}\right)\right)\left(\frac{\pi-\rho}{\sigma \pi}\right) \\
\quad+\nu \int_{-T}^{0} e^{\left(\pi-a_{1}\right) \tau} \delta \Omega \frac{1}{\delta+a_{1}}\left(e^{\left(a_{1}+\delta\right) \tau}-e^{-\left(a_{1}+\delta\right) T}\right) \mathrm{d} \tau \\
=\left(\Omega \frac{1}{\delta+a_{1}}\left(1-e^{-\left(\delta+a_{1}\right) T}\right)-\Omega e^{-\delta T} \frac{1}{a_{1}}\left(1-e^{-a_{1} T}\right)\right)\left(\frac{\pi-\rho}{\sigma \pi}\right) \\
+\nu \frac{\delta \Omega}{\delta+a_{1}}\left(\frac{1}{\pi+\delta}\left(1-e^{-(\pi+\delta) T}\right)-e^{-\left(a_{1}+\delta\right) T} \frac{1}{\pi-a_{1}}\left(1-e^{-\left(\pi-a_{1}\right) T}\right)\right) .
\end{aligned}
$$

We can see that, when $g>0$,

$$
\lim _{a_{1} \rightarrow-\infty} \Sigma\left(a_{1}\right)=+\infty \quad \lim _{a_{1} \rightarrow+\infty} \Sigma\left(a_{1}\right)=0
$$

then there exists a $a_{1}$ such that $\Sigma\left(a_{1}\right)=1$ and than such that $a_{0} e^{a_{1}}$ is the a BGP for all positive $a_{0}$. But from Theorem 3.4 (since we need $b_{0} e^{b_{1} s}-a_{0} e^{a_{1} s}=\Lambda e^{g s}$ for all $s \geq 0$ ) we can deduce that the only possible choice for a BGP is that $a_{1}=b_{1}=g$, then $\Sigma(g)=1$ and $a_{0} e^{g \cdot}$ is a BGP for all positive $a_{0}$. The related state evolution will be $y(s)=b_{0} e^{g s}$ where $b_{0}=\int_{-T}^{0} \Omega\left(e^{\delta s}-e^{-\delta T}\right) a_{0} e^{g s} \mathrm{~d} s$.

\section{Proof of Proposition 3.11}

We have only to use the definitions and results of other sections, to substitute $\bar{\iota}(s)=c e^{g_{0} s}$ $(s \in[-T, 0))$ and to solve the integrals. In particular we have to use (27) for (i), (38) for (ii), (39) and (41) for (iii), and (45) for (iv).

\section{References}

Askenazy, P., Le Van, C., 1999. A model of optimal growth strategy. J. Econ. Theory 85, 24-51.

Bensoussan, A., Da Prato, G., Delfour, M.C., Mitter, S.K., 2007. Representation and control of infinite dimensional systems. Systems \& Control: Foundations \& Applications, Birkhäuser Boston Inc., Boston, MA. second edition.

Boucekkine, R., Licandro, O., Puch, L.A., del Rio, F., 2005. Vintage capital and the dynamics of the AK model. J. Econ. Theory 120, 39-72.

Boucekkine, R., del Rio, F., Martinez, B., 2009. Technological progress, obsolescence, and depreciation. Oxford Econ. Pap. 61, 440-466.

Boucekkine, R., Ruiz-Tamarit, R., 2003. Capital maintenance and investment: Complements or substitutes? J. Econ. 78, 1-28.

Collard, F., Kollintzas, T., 2000. Maintenance, utilization, and depreciation along the business cycle. Centre for Economic Policy Research.

Delfour, M.C., 1986. The linear quadratic optimal control problem with delays in the state and control variables: a state space approch. SIAM J. of Control Optim. 24, 835-883.

Diekmann, O., Van Gils, S., Verduyn Lunel, S., Walther, H., 1995. Delay equations. Springer, Berlin.

Fabbri, G., Gozzi, F., 2008a. Solving optimal growth models with vintage capital: The dynamic programming approach. J. Econ. Theory 143, 331-373. 
Fabbri, G., Gozzi, F., 2008b. Vintage capital in the ak growth model: a dynamic programming approach. extended version. Preprint. http://ideas.repec.org/p/pra/mprapa/2863.html.

Feldstein, M.S., Rothschild, M., 1974. Towards an economic theory of replacement investment. Econometrica , 393-423.

Goetz, R.U., Hritonenko, N., Yatsenko, Y., 2008. The optimal economic lifetime of vintage capital in the presence of operating costs, technological progress, and learning. J. Econ. Dynam. Control 32, 3021-3053.

Hartl, R., 1983. Optimal maintenance and production rates for a machine : A nonlinear economic control problem. J. Econ. Dynam. Control 6, 281-306.

Kalaitzidakis, P., Kalyvitis, S., 2004. On the macroeconomic implications of maintenance in public capital. J. Public Econ. 88, 695-712.

Kalaitzidakis, P., Kalyvitis, S., 2005. New public investment and/or public capital maintenance for growth? the canadian experience. Econ. Inquiry 43, 586-600.

Kalyvitis, S., 2006. Another look at the linear q model: an empirical analysis of aggregate business capital spending with maintenance expenditures. Can. J. Econ. 39, 1282-1315.

Licandro, O., Puch, L., 2000. Capital utilization, maintenance costs and the business cycle. Ann. Econ. Statist., 143-164.

Licandro, O., Puch, L.A., Ruiz-Tamarit, J.R., 2001. Optimal growth under endogenous depreciation, capital utilization and maintenance costs. Investigaciones Econ. 25, 543-559.

McGrattan, E., Schmitz, J., 1999. Maintenance and repair: Too big to ignore. Fed. Reserve Bank Minneapolis Quart. Rev. 23, 2-13.

Nickell, S., 1975. A closer look at replacement investment. J. Econ. Theory 10, 54-88.

Saglam, C., Veliov, V., 2008. Role of Endogenous Vintage Specific Depreciation in the Optimal Behavior of Firms. Int. J. Econ. Theory 4, 381-410.

Vinter, R.B., Kwong, R.H., 1981. The infinite time quadratic control problem for linear system with state control delays: An evolution equation approch. SIAM J. of Control Optim. 19, 139-153.

Whelan, K., 2002. Computers, obsolescence, and productivity. Rev. Econ. Statist. 84, 445-461. 